\title{
An evolutionary disc model of the edge-on galaxy NGC 5907
}

\author{
A. Just ${ }^{1}$, C. Möllenhoff ${ }^{2}$, and A. Borch ${ }^{1}$ \\ 1 Astronomisches Rechen-Institut am ZAH, Mönchhofstraße 12-14, 69120 Heidelberg, Germany \\ e-mail: just@ari.uni-heidelberg.de \\ ${ }^{2}$ Landessternwarte am ZAH, Königsstuhl 12, 69117 Heidelberg, Germany
}

Received 26 May 2006 / Accepted 2 August 2006

ABSTRACT

\begin{abstract}
Context. We present an evolutionary disc model of the edge-on galaxy NGC 5907 based on a continuous star formation history and a continuous dynamical heating of the stellar subpopulations.

Aims. This model explains the disparate two observational facts: 1) the exponential vertical disc structure in the optical and NIR of the non-obscured part of the stellar disc and 2) the FIR/submm luminosity enhanced by about a factor of four near the obscured mid-plane, which requires additional dust and also stellar light to heat the dust component.

Methods. We use multi-band photometry in $U, B, V, R$, and $I$-band combined with radiative transfer through a dust component to simultaneously fit the vertical surface-brightness and colour index profiles in all bands adopting a reasonable star formation history and dynamical heating function. The vertical distribution of the stellar subpopulations are calculated self-consistently in dynamical equilibrium and the intrinsic stellar emissivity is calculated by stellar population synthesis.

Results. The final disc model reproduces the surface-brightness profiles in all bands with a moderately declining star formation rate and a slowly starting heating function for young stars. The total dust mass is $5.7 \times 10^{7} M_{\odot}$ as required from the FIR/submm measurements. Without a recent star burst we find in the midplane an excess of 5.2-, 4.0-, and 3.0-times more stellar light in the $U$-, $B$-, and $V$-band, respectively. The corresponding stellar mass-to-light ratios are 0.91 in $V$ - and 1.0 in $R$-band. The central face-on optical depth in $V$-band is $\tau_{\mathrm{v}}^{\mathrm{f}}=0.81$ and the radial scale length of the dust is $40 \%$ larger than that of the stellar disc.

Conclusions. Evolutionary disc models are a powerful method to understand the vertical structure of edge-on galaxies. Insights into the star formation history and the dynamical evolution of stellar discs can be gained. FIR/submm observations are necessary to restrict the parameter space for the models.
\end{abstract}

Key words. stellar dynamics - galaxies: kinematics and dynamics - galaxies: evolution - galaxies: stellar content galaxies: structure

\section{Introduction}

\subsection{Vertical structure of stellar discs}

The vertical structure of the stellar disc in late-type galaxies is mainly determined by the star formation history combined with the dynamical heating $\sigma(t)$ of the stellar population due to gravitational perturbations. Young stellar subpopulations are confined tightly to the midplane with small velocity dispersion, whereas the older subpopulations are distributed over larger heights due to their higher velocity dispersion. This basic feature is wellknown in the Galaxy (Wielen 1977; Freeman 1991; Edvardsson et al. 1993 e.g.). Dynamical heating leads to an increasing mean age of the population with increasing height above the midplane. This results in an increasing mass-to-light ratio and vertical colour index gradients from blue to red. In principle, these colour index gradients should be directly observable in edge-on galaxies but unfortunately, strong dust extinction and reddening often dominates the vertical colour index distribution. Therefore detailed models are necessary to extract the intrinsic stellar disc properties from multi-colour photometry.

In a series of papers van der Kruit \& Searle (1981, 1982a,b) started to investigate the vertical and radial properties of the surface-brightness profiles of edge-on galaxies. They determined for a number of nearby galaxies (including NGC 5907) the radial scale length, the radial cutoff, and the vertical scale length. They recognised that the shape of the vertical surface-brightness profiles can be well fitted by a $\operatorname{sech}^{2}$-model disregarding the central minimum in the case of strong dust lanes. In recent decades much work has been done on the phenomenological analysis of edge-on galaxies (e.g. Guthrie 1992; Pohlen et al. 2002).

In our Galaxy the stellar subpopulations of the thin disc are not homogeneously mixed. The vertical thickness of the subpopulations increases with age (Wielen \& Fuchs 1988). Wainscoat et al. (1989) observed a similar vertical structure in the edgeon galaxy IC 2531. For the analysis of the intrinsic physical structure of galactic discs we started to investigate the vertical surface-brightness and colour index profiles of edge-on galaxies in order to analyse the age distribution of stellar discs (Wielen et al. 1992; Just et al. 1996). We used evolutionary stellar population models for the intrinsic light distribution and include dust extinction in the radiative transfer for the observable vertical surface-brightness profiles. We found that in edge-on galaxies the vertical colour index gradients above the innermost part, where extinction dominates, are a signature of the changing properties of the stellar population with height above the midplane. De Grijs \& Peletier (2000) also found from a large sample of edge-on galaxies that the stellar population properties in the discs can be seen in the vertical colour index gradients above the extinction features.

For NGC 5907 and other nearby edge-on galaxies Xilouris et al. (1997, 1999) used an extensive radiative transfer code including scattered light to model the two-dimensional light 
distribution of the stellar discs. Using exponential intrinsic luminosity distributions different for each band $(B, V, I$ in the case of NGC 5907) and an exponential disc for the dust component, they determined radial and vertical scale lengths of the star light and the properties of the dust component. The resulting relative low dust mass corresponds to a gas-to-dust ratio of 810 (corrected for the helium contribution). In recent years FIR observations offered a new, more direct way to deduce the dust properties of galaxies. Popescu et al. (2000) developed a sophisticated temperature model of the dust component in edge-on galaxies in order to fit the FIR observations and applied it to NGC 891. With this method Misiriotis et al. (2001) determined for NGC 5907 a dust mass 4 times larger than Xilouris et al. (1999). For the source of the FIR excess they adopted an additional young stellar component in a very thin disc, which is completely hidden by dust extinction in the optical and NIR bands. Recently Stevens et al. (2005) used a two-temperature dust model to fit the IRAS and SCUBA data for a sample of spiral galaxies. For NGC 5907 they determine a dust mass only three times larger than the Xilouris et al. value.

\subsection{Content of this paper}

In this paper we present an evolutionary stellar disc model for NGC 5907, which explains both observational results: 1) The exponential vertical profiles in all optical bands are reproduced by the outer stellar disc dominated by the older thin disc population and are no longer just a set of independent fit functions in each band as in Xilouris et al. (1999). 2) The large amount of dust determined from FIR/submm observations by Misiriotis et al. (2001) exceeding the dust component found in Xilouris et al. by a factor of four is necessary in our model to obscure the additional light of the young stellar subpopulations with low mass-to-light ratio near the midplane of the disc. There is no additional recent star burst required to explain the additional star light needed for the dust heating.

The basis of our disc model is a vertical cut with a selfconsistent detailed distribution of stellar subpopulations. The vertical distribution of the stars is determined by the dynamical equilibrium of the stellar subpopulations described by the star formation history of the disc and by the dynamical heating function (measured by the increasing velocity dispersion). The gravitational forces of the gas component and the dark matter halo are included. The intrinsic stellar light distribution is calculated from population synthesis models of simple stellar populations (SSP). The resulting vertical profile is then extended to an exponential disc up to the cutoff radius $R_{\max }$ by scaling the surface densities with constant scale heights. The intrinsic structure is not changed as a function of radius. We compute the observable vertical surface-brightness and colour index profiles by radiative transfer calculations with an exponential dust component, where the inclination of the disc with respect to the line of sight is fitted.

The parameters of the basic vertical profile are chosen to match all constraints at some fixed radius $R_{0}$ of the disc, where the disc is dominating the light and the bulge can be neglected. At that radius we fix the relative contribution of the gas and dark matter component $Q_{\mathrm{g}}=\Sigma_{\mathrm{g}} / \Sigma_{\text {tot }}$ and $Q_{\mathrm{h}}=\Sigma_{\mathrm{h}} / \Sigma_{\text {tot }}$ to the total surface density $\Sigma_{\text {tot }}$ up to a maximum height $z_{\text {max }}$ above the midplane. We will use $R_{0}=10 \mathrm{kpc} \approx 2 R_{\mathrm{s}}$ with radial scale length $R_{\mathrm{S}}$ of the stellar disc, which is near the maximum of $\Sigma_{\mathrm{d}} / \Sigma_{\mathrm{h}}$ in the case of an isothermal halo. The scale height of the gas component relative to the effective scale height of the stars $z_{\mathrm{g}} / z_{0}$ and $s_{\mathrm{h}}=\sigma_{\mathrm{h}} / \sigma_{\mathrm{e}}$, the ratio of the velocity dispersion of the dark

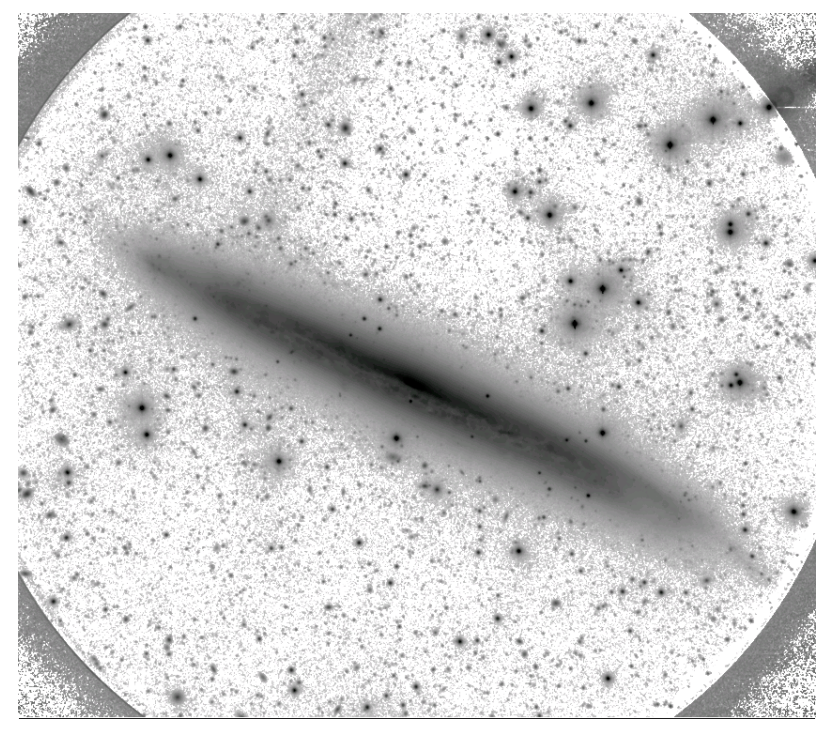

Fig. 1. Deep $R$ image of NGC 5907, sum of 4 exposures. The field of view is $15 \times 13.25$ arcmin. $\mathrm{N}$ is top, $\mathrm{E}$ is left. (cf. Sect. 5).

matter halo and the maximum velocity dispersion of the stars are also determined at $R_{0}$.

In an iterative process the crucial fitting functions $\operatorname{SFR}\left(t_{\mathrm{a}}-t\right)$ and $\sigma(t)$ are optimised together with the global parameters: inclination $i$, radial scale length $R_{\mathrm{s}}$, effective scale height $z_{\mathrm{s}}$, dust distribution, and total stellar and dust mass. For comparison with observations we use a series of vertical surface-brightness (and colour index) profiles parallel to the minor axes in $U, B, V, R$, $I$ bands, which we obtained from corresponding deep photometric images of NGC 5907. The same type of intrinsic disc model was also successfully applied to the solar neighbourhood in order to analyse the connection between the local velocity distribution functions of the main sequence stars from the Catalogue of Nearby Stars (CNS4, Jahreiss \& Wielen 1997) and the vertical density profiles of these stars (see Just 2001, 2002, 2003).

In Sect. 2 we collect the basic parameters of NGC 5907, which are necessary for the basic scaling of our disc model to the observational data. In Sect. 3 we describe the building blocks of our disc model. Section 4 gives the iteration procedure with details on the scaling method, radiative transfer and the multicolour analysis. In Sect. 5 our observations and the derivation of vertical colour index and surface-brightness profiles are presented. Figure 1 shows a deep $R$ image (cf Sect. 5 as an example of for the high quality of data. In Sect. 6 we show the final comparison of the profiles, give the global parameters of our best model and discuss in detail the intrinsic structure of the stellar disc. A discussion of some crucial aspects follow in Sect. 7. Section 8 contains the concluding remarks.

\section{Basic parameters of NGC 5907}

Here we discuss the basic scaling parameters of NGC 5907, namely distance, radial and vertical scale length and inclination of the stellar disc, and the masses of the stellar, gaseous and dark matter component.

\subsection{Distance to NGC 5907}

There is some uncertainty in the distance determination of nearby galaxies from the Hubble expansion, because the peculiar motion of the galaxies is not negligible. In this work we use 
a distance of $D=11 \mathrm{Mpc}$ with $V_{\mathrm{GSR}}=817 \mathrm{~km} \mathrm{~s}^{-1}$ from the RC3 catalogue (de Vaucouleurs et al. 1991) and a Hubble constant of $H_{0}=75 \mathrm{~km} \mathrm{~s}^{-1} \mathrm{Mpc}^{-1}$. The resulting scale conversion is $1^{\prime \prime}=53.3 \mathrm{pc}$ and the distance modulus is $\Delta m=30.2 \mathrm{mag}$.

Another distance determination for NGC 5907 using the Tully-Fisher relation leads to a slightly larger distance of $D=$ 11.6 or $12.0 \mathrm{Mpc}$ (Sofue 1996, 1997). In this method the main uncertainty arises from the conversion of the edge-on luminosity to a face-on luminosity. Zepf et al. (2000) used a distance of $D=13.5 \pm 2.1 \mathrm{Mpc}$ from a combination of the $R$-band TullyFischer relation with a peculiar motion model and discussed also (due to the lack of resolved giant stars in the outer bulge region from HST observations) the possibility of a significantly larger distance.

\subsection{Disc parameters}

For the outer parts of the disc, where extinction is not significant, the brightness distribution of the disc of NGC 5907 can be modelled by exponential profiles in the vertical and radial direction. The radial scale length increases from $3.7 \mathrm{kpc}$ in $H$ band (Barneby \& Thronson 1994) to more than $5 \mathrm{kpc}$ in the blue (van der Kruit \& Searle 1982a: $5.7 \mathrm{kpc}$ in $J$-band, Xilouris et al. 1999: $5.02 \mathrm{kpc}$ in $B$-band). Van der Kruit \& Searle also determined the cutoff radius of the exponential disc at $R_{\max }=$ $19.3 \mathrm{kpc}$, which has some influence on the inner parts of the radial surface-brightness profiles due to projection effects. The cutoff was not included in the model of Xilouris et al. (1999) leading to an underestimation of the intrinsic scale length.

The effective scale height of the disc is approximately independent of radius, but depends strongly on the model used, i.e. additional components like thick disc, bulge and also on the radial profile for edge-on galaxies. From moderately deep photometry van der Kruit \& Searle found $z_{\mathrm{s}}=410 \mathrm{pc}$ in $\mathrm{J} / \mathrm{band}$ and Xilouris et al. $z_{\mathrm{s}}=340 \mathrm{pc}$ in $B$-band, and Barneby \& Thronson $z_{\mathrm{s}}=320 \mathrm{pc}$ in $H$-band, which are relatively small values. With a One-disc-model for the very deep $R$-band photometry Morrison et al. (1994) determined a scale height of $z_{\mathrm{s}}=467 \mathrm{pc}$.

From the shape of the outer isophotes van der Kruit \& Searle found an inclination of $i=87.0^{\circ}$, which is widely used as a standard value. Xilouris et al. determined independently a value of $i=87.2^{\circ}$. Morrison et al. (1994) observed a stellar warp in the outer parts of the disc. Therefore the intrinsic inclination may be even higher, since the warp smears out the isophotes additively.

Since in our disc model we assume only one stellar disc for all five observed bands, we redetermine the radial and vertical scale length, the cutoff radius and the inclination in the fitting procedure.

\subsection{Masses}

For the construction of the disc model we need the relative surface densities of the different components. The scaling to the absolute values is then a result of the surface-brightness profile fitting (cf. Sect. 4.3), and the total masses of the stellar and gaseous components follow from the radial extrapolation to exponential discs. We use the total hydrogen mass $M_{\mathrm{H}}$ and convert it to the total gas mass $M_{\mathrm{g}}$ which will be used for the determination of the gravitational force and to calculate the gas-to-dust ratio $F$. For the dark matter halo we need only a reliable estimate of the local density in the disc.

\subsubsection{Gas masses}

The atomic gas mass is determined from the $21 \mathrm{~cm}$ luminosity. We use the standard value

$M_{\mathrm{HI}}=6.9 \times 10^{9} M_{\odot}$

(Dumke et al. 1997; Stevens et al. 2005). The molecular gas mass is much more uncertain. It is derived by converting CO measurements using the conversion factor $X$, which gives the corresponding $\mathrm{H}_{2}$ mass. Dumke et al. (1997) determined individually for the galaxy NGC 5907 a reduced conversion factor $X$ yielding $M_{\mathrm{H}_{2}}=0.9 \times 10^{9} M_{\odot}$. The standard $X$-value would have led to $1.8 \times 10^{9} M_{\odot}$, which demonstrates the intrinsic uncertainty of the molecular gas mass determination. We use the molecular gas mass redetermined by Stevens et al. (2005) of

$M_{\mathrm{H}_{2}}=1.7 \times 10^{9} M_{\odot}$

(rescaled to the distance of $D=11 \mathrm{Mpc}$ ). For the contribution of He to the total gas mass we add $40 \%$ to the total hydrogen mass $M_{\mathrm{H}}=M_{\mathrm{HI}}+M_{\mathrm{H}_{2}}$. This leads to the total gas mass of

$M_{\mathrm{g}}=1.4 M_{\mathrm{H}}=1.2 \times 10^{10} M_{\odot} \quad$ with

$M_{\mathrm{H}}=M_{\mathrm{HI}}+M_{\mathrm{H}_{2}}=8.6 \times 10^{9} M_{\odot}$.

The radial distribution of $\mathrm{HI}$ is flatter and more extended than the stellar light distribution and is not exponential. The CO distribution is much more confined to the inner parts than that of HI (Dumke et al. 1997). For simplicity we will also adopt for the scaling of the gas fraction an exponential disc, but allow for a scale length larger than the radial scale length of the stellar disc. We will use the same spatial distribution as for the dust component, which is determined by our fitting procedure.

\subsubsection{Stellar and dark matter masses}

The determination of the stellar disc and dark matter (DM) halo mass from the rotation curve is very uncertain due to the wellknown disc-halo degeneracy. Sackett et al. (1994) constructed three-component mass models (HI, stellar disc, dark matter halo) to fit the HI-rotation curve of Sancisi \& van Albada (1987). They found equally good fits with disc mass-to-light ratios of 1,2 , and 4 in the $R$-band. The models of Sackett et al. with $M / L=1$ and $M / L=2$ yield a range of surface density ratios of stellar disc and halo of

$$
\left.\frac{\Sigma_{\mathrm{s}}}{\Sigma_{\mathrm{h}}}\right|_{R_{0}}=2 \ldots 5 \text { with } R_{0}=10 \mathrm{kpc}, z_{\max }=5.1 \mathrm{kpc}
$$

Typical mass-to-light ratios of stellar discs in the $V$-band are of order unity and are dependent on the average age of the population. In the solar neighbourhood we have $M / L_{\mathrm{V}}=0.78$ and the extrapolation to the solar cylinder is $M / L_{\mathrm{V}} \approx 1.4$ for the mass-to-light ratio of the surface density (from CNS4 data, Jahreiss \& Wielen 1997). Our final model yields $M / L_{\mathrm{V}}=0.9$ and $M / L_{\mathrm{R}}=1.0$. Therefore we will use the $M / L_{\mathrm{R}}=1$ model of Sackett et al. (1994) for the scaling of the DM halo.

Sofue (1996) presented the joint rotation curve of CO and $\mathrm{HI}$, which shows in $\mathrm{HI}$ a slightly higher and more pronounced maximum, which is harder to reconstruct with a low mass disc of $M / L_{\mathrm{R}}<2$. From $H$-band photometry Barneby \& Thronson (1994) investigate mass models including a flattened bulge and determined a small bulge with scaling radius $0.23 \mathrm{kpc}$ and total mass of $M_{\mathrm{B}}=9 \times 10^{9} M_{\odot}$. The CO rotation curve of Sofue (1996) confirms the kink produced by the bulge at 
$V_{\mathrm{c}}(R=1 \mathrm{kpc}) \approx 200 \mathrm{~km} \mathrm{~s}^{-1}$ of model b) in Barneby \& Thronson (1994). As a consequence the pollution of the disc luminosity with bulge light at radial distances larger than $3 \mathrm{kpc}$ is very small. Therefore we decided to neglect the bulge component in our analysis in order to keep the number of free parameters small.

\subsubsection{Dust mass and extinction}

Our aim is to construct a disc model with a dust component, which has a mass comparable to

$M_{\mathrm{d}}=6 \times 10^{7} M_{\odot}$,

the value determined by Misiriotis et al. (2001). We use the standard extinction law of Rieke \& Lebofsky (1985), which was confirmed by Xilouris et al. (1999) for NGC 5907 and which also goes into the conversion factor of extinction to dust mass. In our model we determine the spatial distribution of extinction $A_{\mathrm{V}}(R, z)$ (see Eq. (24)). This will be converted to the dust mass distribution by

$\rho_{\mathrm{d}}=0.175 \times 10^{-3} A_{\mathrm{V}} M_{\odot} \mathrm{pc}^{-3}$

with $A_{\mathrm{V}}$ in $[\mathrm{mag} / \mathrm{kpc}]$. This is the same conversion factor as used by Xilouris et al. (1999) and Misiriotis et al. (2001).

\section{Building blocks of the disc model}

The aim of this work is to construct a physical model of the stellar disc in order to reproduce the vertical surface-brightness and colour index profiles from $U, B, V, R$, and $I$-band observations. Therefore we pay much attention to the internal structure of the stellar disc. The gas and dust component and the dark matter halo are modelled in a simple way. The bulge contribution is neglected, because the number of free parameters and fitting functions would be approximately doubled with very small effect on the parameters of the disc. A thick disc component is excluded by very deep photometry and the faint stellar halo is below our observational limit (Morrison et al 1994). We apply our model to those regions of the disc where the thin disc strongly dominates.

In this section we describe the different ingredients necessary to construct the disc model and compute their intrinsic properties. The projection onto the sky and the fitting procedure is given in Sect. 4.

\subsection{Self-gravitating disc}

The backbone of the disc is a self-gravitating vertical disc profile including the gas component in the thin disc approximation. In this approximation the Poisson-equation is one-dimensional

$\frac{\mathrm{d}^{2} \Phi_{\text {self }}}{\mathrm{d} z^{2}}=4 \pi G \rho(z)$,

where $\rho(z)$ is the self-gravitating density and $\Phi_{\text {self }}(z)$ is the corresponding potential. Since we want to construct the disc in dynamical equilibrium, the density of the sub-components will be given as a function of the total potential $\rho_{\mathrm{j}}(\Phi)$ and not of height $z$. In the case of a purely self-gravitating thin disc (with $\Phi_{\text {self }}=\Phi$, i.e. no external potential) the Poisson equation can be integrated leading to

$\left(\frac{\mathrm{d} \Phi}{\mathrm{d} z}\right)^{2}=8 \pi G \int_{0}^{\Phi} \rho\left(\Phi^{\prime}\right) \mathrm{d} \Phi^{\prime}$.
Then the vertical distribution is given by the implicit function $z(\Phi)$ via direct integration

$z=\int_{0}^{\Phi} \mathrm{d} \Phi^{\prime}\left[8 \pi G \int_{0}^{\Phi^{\prime}} \rho\left(\Phi^{\prime \prime}\right) \mathrm{d} \Phi^{\prime \prime}\right]^{-1 / 2}$

If an external potential is included, an iteration process is necessary to solve for the vertical distribution. In order to avoid the iteration at that point, we model all gravitational components by a thin disc approximation. We include in the total potential $\Phi$ the stellar component $\Phi_{\mathrm{s}}$, the gas component $\Phi_{\mathrm{g}}$ and the dark matter halo contribution $\Phi_{\mathrm{h}}$

$\Phi(z)=\Phi_{\mathrm{s}}(z)+\Phi_{\mathrm{g}}(z)+\Phi_{\mathrm{h}}(z)$.

In order to obtain the force of a spherical halo correctly in the thin disc approximation, we use a special approximation (see Sect. 3.4). The relative contribution of the stellar, the gaseous, and the DM-component to the surface density (up to $|z|=z_{\max }$ ) are given by the input parameters $Q_{\mathrm{s}}, Q_{\mathrm{g}}, Q_{\mathrm{h}}$.

\subsection{Stellar disc}

We assume that the disc of NGC 5907 is, like the thin disc of the Milky Way, composed of a sequence of stellar subpopulations with increasing vertical scale height with age. This can be parametrised by the star formation history and the dynamical heating function. For convenience we use here the normalisation to the central profile at $R=0$. The stellar component is composed of a sequence of isothermal subpopulations characterised by the IMF, the chemical enrichment $[\mathrm{Fe} / \mathrm{H}](t)$, the star formation history $\operatorname{SFR}\left(t_{\mathrm{a}}-t\right)$, and the dynamical evolution described by the vertical velocity dispersion $\sigma(t)$. Here $t$ is the age of the subpopulation running back in time from the present time $t_{\mathrm{a}}=12 \mathrm{Gyr}$ (which is the adopted age of the disc). We include mass loss due to stellar evolution and retain the stellar-dynamical mass fraction $g(t)$ (stars + remnants) only. The mass lost by stellar winds, supernovae and planetary nebulae is mixed implicitly to the gas component.

With the Jeans equation the vertical distribution of each isothermal subpopulation is given by

$\rho_{\mathrm{s}, \mathrm{j}}(z)=\rho_{\mathrm{s} 0, \mathrm{j}} \exp \left(\frac{-\Phi(z)}{\sigma^{2}\left(t_{\mathrm{j}}\right)}\right)$,

where $\rho_{\mathrm{s}, \mathrm{j}}$ is actually a "density rate", the density per age bin. The connection to the $S F R$ is given by the integral over $z$

$g\left(t_{\mathrm{j}}\right) \operatorname{SFR}\left(t_{\mathrm{a}}-t_{\mathrm{j}}\right)=\int_{-\infty}^{\infty} \rho_{\mathrm{s}, \mathrm{j}}(z) \mathrm{d} z$.

The (half-)thickness $h_{\mathrm{p}}\left(t_{\mathrm{j}}\right)$ is defined by the midplane density $\rho_{\mathrm{s} 0, \mathrm{j}}$ through

$\rho_{\mathrm{s} 0, \mathrm{j}}=\frac{\operatorname{SFR}\left(t_{\mathrm{a}}-t_{\mathrm{j}}\right)}{2 h_{\mathrm{p}}\left(t_{\mathrm{j}}\right)}$.

The total stellar density is

$\rho_{\mathrm{s}}(z)=\int_{0}^{t_{\mathrm{a}}} \rho_{\mathrm{s}, \mathrm{j}}(z) \mathrm{d} t$

which then determines the potential $\Phi_{\mathrm{s}}(z)$ via the Poisson Eq. (8). The stellar surface density $\Sigma_{\mathrm{s}}$ is connected to the integrated star formation $S_{0}$ by the effective stellar-dynamical fraction $g_{\text {eff }}$

$\Sigma_{\mathrm{s}}=\int \rho_{\mathrm{s}}(z) \mathrm{d} z=g_{\mathrm{eff}} S_{0}$ 
with

$S_{0}=\int S F R \mathrm{~d} t$ and $g_{\mathrm{eff}}=\frac{\int g(t) S F R\left(t_{\mathrm{a}}-t\right) \mathrm{d} t}{S_{0}}$.

The effective scale height $z_{\mathrm{s}}$ of the stellar disc is connected to the maximum velocity dispersion $\sigma_{\mathrm{e}}$ of the subpopulations and the total surface density $\Sigma_{\text {tot }}$ via

$z_{\mathrm{s}}=C_{z} z_{\mathrm{e}}=C_{z} \frac{\sigma_{\mathrm{e}}^{2}}{2 \pi G \Sigma_{\mathrm{tot}}}$,

where $z_{\mathrm{e}}$ is the scale height of an isothermal component above a disc with total surface density $\Sigma_{\text {tot }}$. The shape correction factor $C_{z}$ is of order unity and is determined at $z=(3 \pm 0.5) z_{\mathrm{e}}$.

The metalicity $Z$ affects the stellar lifetimes, luminosities and colours of the subpopulations. $Z$ shows a vertical gradient, since the mean age of the stellar population is correlated with the vertical distribution. In order to account for the systematic influence of the metal enrichment we adopt a moderate metal enrichment similar to the solar neighbourhood (Twarog 1980; Edvardsson et al. 1993). We use the enrichment law for oxygen

$Z=z_{0}+Y \ln \left(1+a \frac{t}{t_{\mathrm{a}}}\right) \quad$ with $\quad Y=\frac{Z_{\mathrm{p}}-z_{0}}{\ln (1+a)}$

with $Z$ normalised to the solar abundance and the conversion law $[\mathrm{Fe} / \mathrm{H}]=2[\mathrm{O} / \mathrm{H}]$ as a simple analytic description to account for the enrichment delay by SN1a. This model is borrowed from a closed-box model with an $n=2$ Schmidt-law for the star formation (Lynden-Bell 1975; Just et al. 1996). We use $a=5$, initial and present metalicity $z_{0}=0.4, Z_{\mathrm{p}}=1.13$ corresponding to $[\mathrm{Fe} / \mathrm{H}]=-0.8$ and $[\mathrm{Fe} / \mathrm{H}]=0.1$ with solar metalicity $Z_{\odot}=0.018$, respectively (see Fig. 2).

In the fitting procedure (Sect. 4.1) a pair of star formation history and heating function is selected to derive the intrinsic structure of the disc. The star formation history and heating function of the final model are shown in Fig. 2.

\subsection{Gas and dust component}

For the gas and dust component we use simple models to account for the gravitation of the gas and the extinction of the dust. In the radial direction we adopt an exponential profile with scale length $R_{\mathrm{d}}=q_{\mathrm{d}} R_{\mathrm{s}}$, where we allow for a difference in the scale length of the stellar component by the factor $q_{\mathrm{d}}$. For the extinction by the dust component we use a simple exponential profile with vertical scale height $z_{\mathrm{d}}$.

The vertical profile of the gas component, which is used for the gravitational force of the gas, is constructed dynamically like the stellar component. The gas distribution is modeled by distributing the gas with a constant rate over the velocity dispersion range $\sigma(t)$ of the young stars up to a maximum age $t_{\mathrm{g}}$. By varying $t_{\mathrm{g}}$ we force the scale height of the gas $z_{\mathrm{g}}$ to the same value as that of the dust component $z_{\mathrm{d}}$. The surface density of the gas is related to the stellar surface density by the ratio $Q_{\mathrm{g}} / Q_{\mathrm{s}}=\Sigma_{\mathrm{g}} / \Sigma_{\mathrm{s}}$, which is determined at the reference radius $R_{0}$ in the fitting procedure (cf. Sect. 4).

\subsection{Dark matter halo}

The halo does not fulfil the thin disc approximation. For a spherical halo we get the vertical component of the force to lowest order from

$\frac{\mathrm{d} \Phi_{\mathrm{h}}}{\mathrm{d} z}=\frac{G M_{\mathrm{R}}}{R^{2}} \frac{z}{R}$
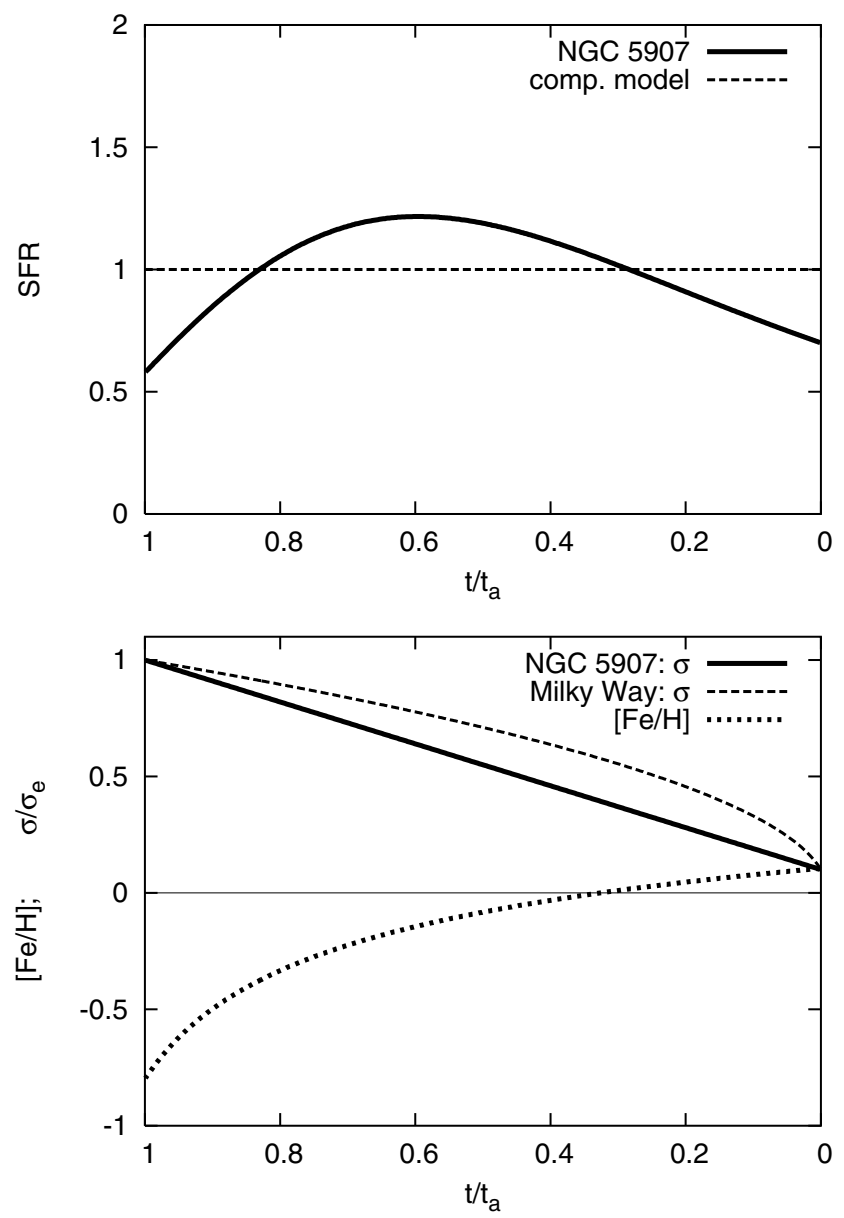

Fig. 2. The upper panel shows the normalised $S F R / S_{0}$ as function of normalised age for the final model of NGC 5907. The dashed line is the constant SFR of a comparison model. The lower panel gives the corresponding heating function $\sigma(t) / \sigma_{\mathrm{e}}$ normalised to the final velocity dispersion $\sigma_{\mathrm{e}}$ (full line) and the chemical enrichment (dotted line). The dashed line is the heating function of the solar neighbourhood used for the comparison model.

with $r^{2}=R^{2}+z^{2}$ and $M_{\mathrm{R}}$ is the enclosed halo mass inside radius $R$. Comparing this with the one-dimensional Poisson equation from the thin disc approximation (Eq. (8) integrated over $z$ near the midplane to lowest order for small $z$ )

$\frac{\mathrm{d} \Phi}{\mathrm{d} z}=4 \pi G \rho_{0} z$

we should use for the local halo density

$\rho_{\mathrm{h} 0}=\frac{M_{\mathrm{R}}}{4 \pi R^{3}}$

which exactly corresponds to the singular isothermal sphere. Therefore we can use the thin disc approximation also for the halo, if we use the local halo density $\rho_{\mathrm{h} 0}$ and the halo velocity dispersion $\sigma_{\mathrm{h}}$ estimated from the rotation curve by adopting an isothermal spherical halo. The effect of a cored halo, anisotropy and flattening is neglected here. For other halo profiles correction factors would be necessary introducing some inconsistency in the halo profile description and leading to a different local halo density. The latter would be more important, because the effect of the halo potential on the disc is stronger than the adiabatic contraction of the halo in the disc potential. 

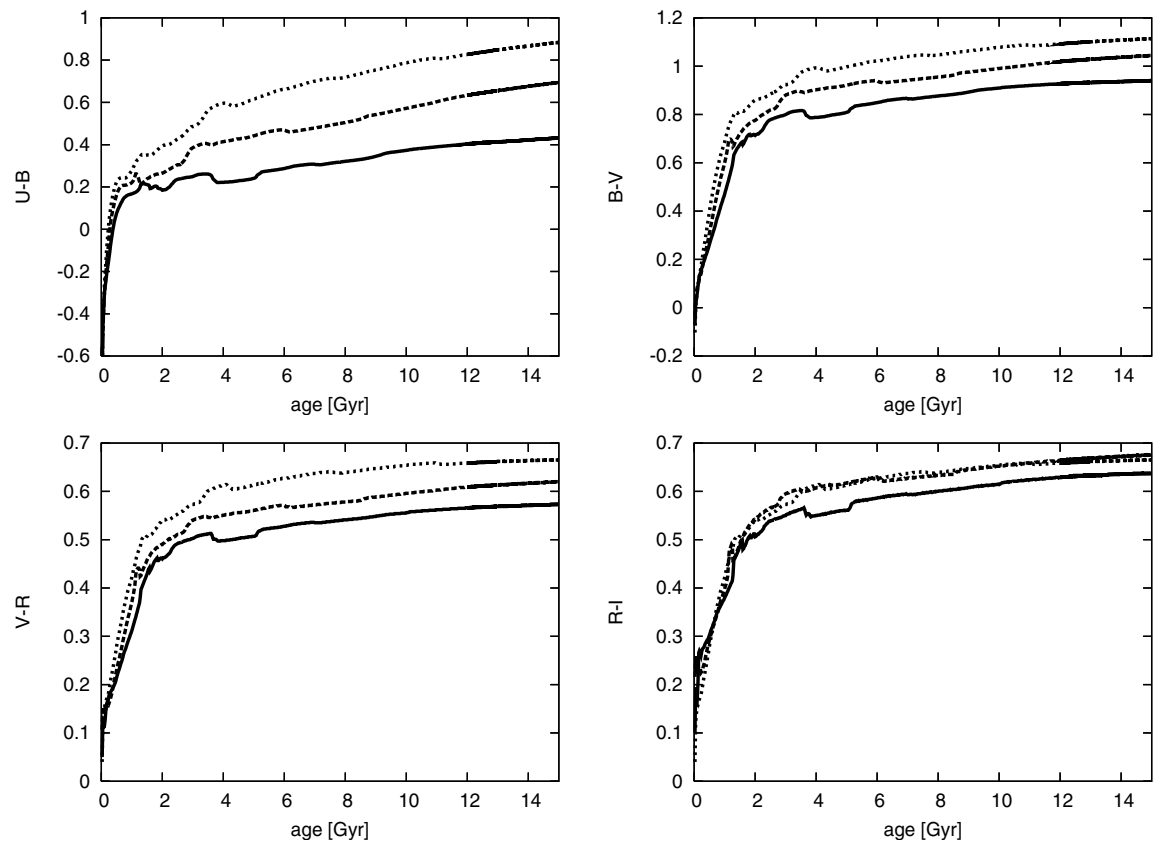

Fig. 3. Colour index evolution of single stellar populations modelled with the PEGASE code (Fioc \& Rocca-Volmerange 1997). These SSPs are created in a star-burst lasting for 25 Myr. We use a Scalo IMF (Scalo 1986). Three different metalicities are shown in the plots: solid: $Z=0.008$, long-dashed: $Z=0.02$, short-dashed: $Z=0.04$.

\subsection{Radial structure}

The parameters of the self-consistent vertical profile described in the last section are determined at a scaling radius $R_{0}=10 \mathrm{kpc}$. The determination of the disc properties at $R_{0}$ are described in Sect. 4.1. For the radial structure of the disc we use a simple exponential extension up to a cutoff radius $R_{\max }$. We do not account for the radial variation of intrinsic disc properties, which are 1) the star formation history or metalicity to fit the radial colour index gradients; 2) the increasing dark matter mass fraction with radius; 3 ) the possible variation of the star/gas surface density ratio due to different radial scale lengths; 4) the breakdown of the thin disc approximation in the innermost part of the disc; 5) the bulge potential and luminosity in the inner region.

The stellar disc model is then given by

$\rho_{\mathrm{S}}(R, z)=\rho_{\mathrm{s}}(z) \exp \left(-R / R_{\mathrm{S}}\right) \quad$ for $\quad R<R_{\max }$

with radial scale length $R_{\mathrm{s}}$, cutoff radius $R_{\max }$ and constant scale height $z_{\mathrm{s}}$.

For the gas and dust component we allow for a different radial scale length $R_{\mathrm{g}}=R_{\mathrm{d}}=q_{\mathrm{d}} R_{\mathrm{s}}$ with scaling factor $q_{\mathrm{d}}$. For the conversion of total masses to density distributions we do not apply a cutoff radius. The corresponding distribution of extinction is

$A_{\mathrm{V}}(R, z)=A_{\mathrm{V}, 0} \exp \left(\frac{-R}{q_{\mathrm{d}} R_{\mathrm{S}}}-\frac{|z|}{z_{\mathrm{d}}}\right)$

with central extinction coefficient $A_{\mathrm{V}, 0}$.

\subsection{Stellar population synthesis}

The intrinsic luminosities in the different bands are determined by stellar luminosity synthesis. We use the stellar population synthesis code PEGASE (Fioc \& Rocca-Volmerange 1997) to produce "pseudo" simple stellar populations (SSPs), which are used for computing the intrinsic luminosity distribution of the stellar disc. This means that the PEGASE code is used to calculate the integrated colour indices for a stellar population created in a single star-burst at different time-steps. These SSPs are then used to assemble a stellar population with a given star formation history, in the sense that the star formation history is assembled by a series of star-bursts. Since we do the assembling of SSPs ourselves, we have to take into account our own treatment of chemical enrichment.

Our "pseudo" SSPs are modeled by a constant star formation rate with a duration of $25 \mathrm{Myr}$. As a input parameter for the PEGASE code we use a fixed Scalo-like IMF (Scalo 1986) given by

$$
\begin{aligned}
\mathrm{d} N \propto M^{-\alpha} \mathrm{d} M & \\
\alpha & =\left\{\begin{array}{lc}
1.25 & 0.08 \leq M / M_{\odot}<1 \\
2.35 \text { for } & 1 \leq M / M_{\odot}<2 \\
3.0 & 2 \leq M / M_{\odot}<100
\end{array}\right.
\end{aligned}
$$

The stellar mass of this population is normalised to $10^{10}$ solar masses. We use the PEGASE code to produce flux tables of broadband colours at linear spaced time-steps of $25 \mathrm{Myr}$. This is done for the three different metalicities $(Z=0.008 ; 0.02 ; 0.04)$, which are the input parameters into our code, where the chemical enrichment is approximated by linear interpolation of populations with these fixed metalicities.

Figure 3 shows the colour index evolution of our SSPs for the three different metalicities.

The normalised luminosities in different broadband filters are shown in Fig. 4, and the mass-to-light ratios are shown in Fig. 5. These SSP lookup-tables are used to assemble the total fluxes and stellar masses for a given star formation history. In Fig. 5 the fraction of initial mass in luminous stars, remnants and the sum of both, which goes into the stellar disc mass and the stellar mass-to-light ratio, is plotted for solar metalicity. 


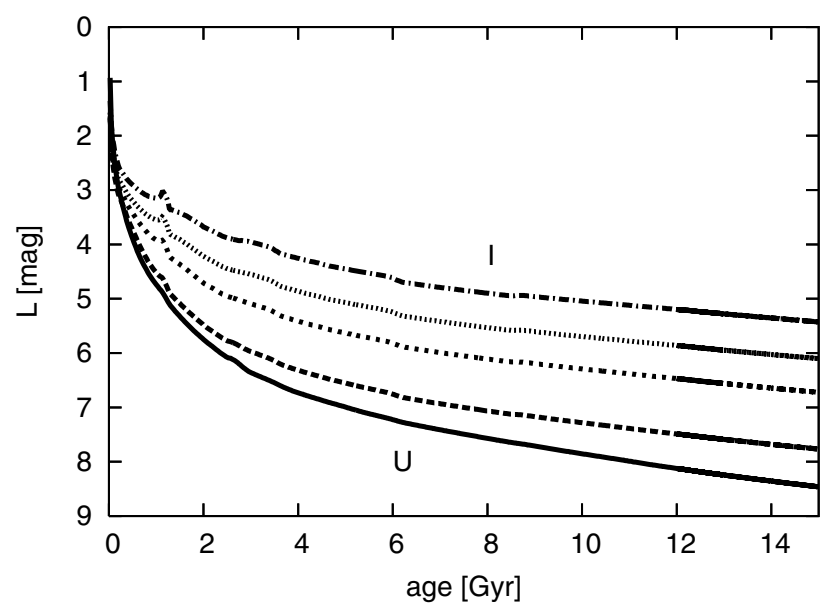

Fig. 4. Luminosities of single stellar populations modelled with the PEGASE code with a Scalo IMF (Scalo 1986) for solar metalicities. The luminosities are normalised to 1 solar mass. The different lines show different broadband filters: solid: $U$, long-dashed: $B$, short-dashed: $V$, dotted: $R$, dot-dashed: $I$.

\section{Radiative transfer and the fitting procedure}

Here we describe the computation of the surface density profiles and explain the main effects on the vertical profiles due to the variation of the disc parameters.

The disc model is derived by performing a three-step iteration cycle until good agreement of the model profiles with the observed surface-brightness and colour index profiles is reached. In the first step the normalised vertical disc profile is derived by adopting a star formation history and dynamical heating function combined with the relative contribution of gas and DM halo component. The intrinsic vertical profile is constructed to fit the galaxy properties at the scaling radius $R_{0}$. In the second step the model is scaled with central surface density, radial and vertical scale length of the stellar disc. Then the inclination with respect to the sky and the dust parameter are chosen to calculate the surface brightness distribution of vertical cuts parallel to the minor axis with a radiative transfer code. The geometrical parameters are varied until a good match to the set of $V$-band profiles is reached. Here the scaling of the gas and halo properties are also corrected iteratively. In the third step a multi-colour analysis is performed to analyse the deviations of the model to the complete set of observed surface-brightness and colour index profiles. These systematic deviations are used to estimate corrections for the star formation history and the heating function for an improved model. In the following these three steps are described in more detail.

\subsection{The normalised vertical profiles}

We start with a pair $\operatorname{SFR}\left(t_{\mathrm{a}}-t\right)$ and $\sigma(t)$ expected for a Sc-galaxy from a basis set of normalised template functions of different types. For the calculation of the self-consistent profile we need additionally the parameters of the gas and halo component normalised to the stellar parameter. These are

1) the fractional surface densities of gas $Q_{\mathrm{g}}$ and DM halo $Q_{\mathrm{h}}$ (leading to $Q_{\mathrm{s}}=1-Q_{\mathrm{g}}-Q_{\mathrm{h}}$ for the stars);

2) the gas scale height normalised to the scale height of the stars $z_{\mathrm{d}} / z_{\mathrm{s}}$ determined implicitly by the maximum "age" of the gas $t_{\mathrm{g}}$;
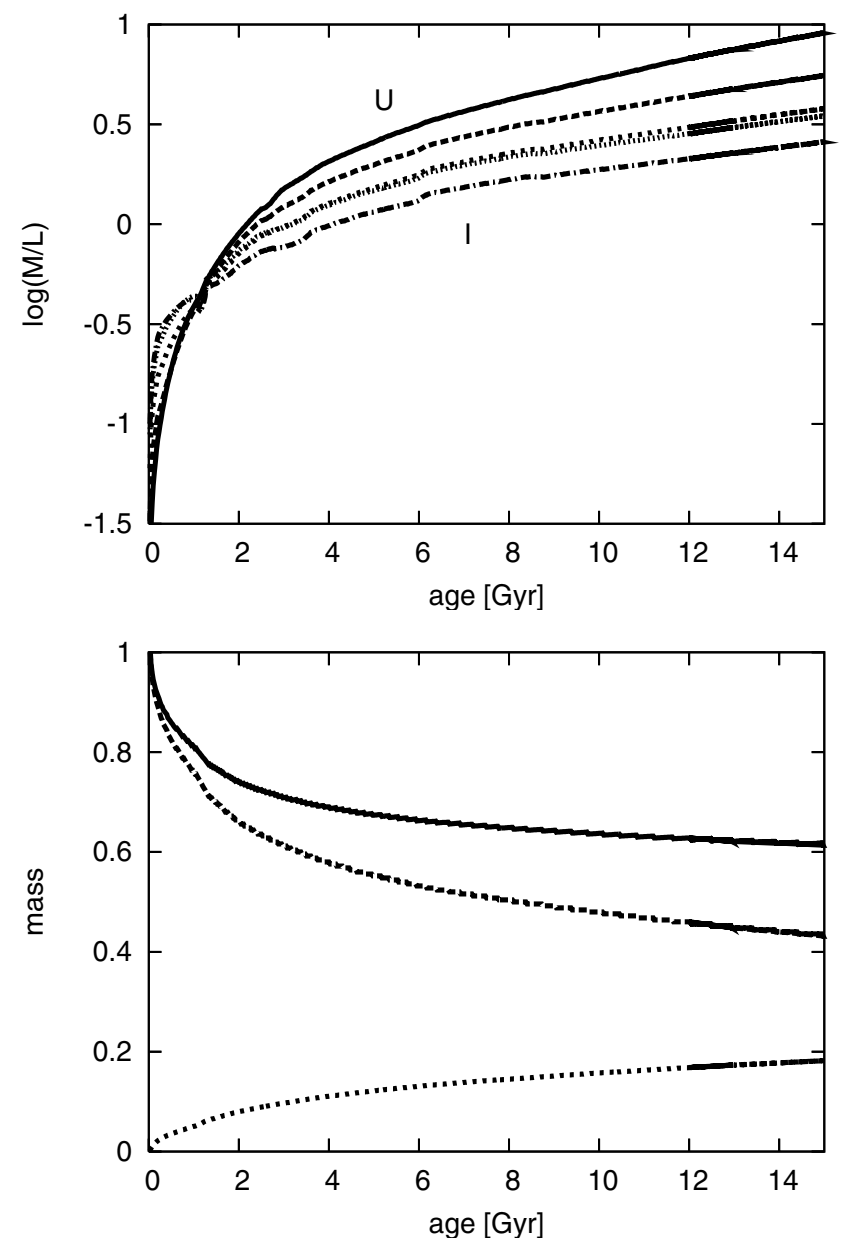

Fig. 5. Evolution of the stellar mass-to-light ratios (including remnants) and of the mass of single stellar populations modelled with the PEGASE code with a Scalo IMF (Scalo 1986). The upper plot shows the M/L evolutions of a solar metalicity SSP in different broadband filters: solid: $U$, long-dashed: $B$, short-dashed: $V$, dotted: $R$, dot-dashed: $I$. We use the solar luminosities $U=5.54, B=5.38, V=4.75, R=4.22$, $I=3.87$. The lower plot shows the mass evolution of the stellar population, normalised to its initial mass. The solid line shows the total stellar mass, and the other lines its two components separately: long-dashed: luminous stars, short-dashed: stellar remnants.

3) the velocity dispersion of the DM halo normalised to the maximum velocity dispersion of the stars $s_{\mathrm{h}}=\sigma_{\mathrm{h}} / \sigma_{\mathrm{e}}$.

Since $Q_{\mathrm{g}}, Q_{\mathrm{h}}, s_{\mathrm{h}}$, and $t_{\mathrm{g}}$ depend on the final scaling of the stellar disc to the observations, they must be corrected iteratively. To fix the vertical profile we use the disc properties at the scaling radius $R_{0}=10 \mathrm{kpc}$. For the gas component the surface density is derived from the total gas mass using the adopted radial scale length. The maximum "age" $t_{\mathrm{g}}$ is chosen to match the vertical scale length of the dust $z_{\mathrm{g}}=z_{\mathrm{d}}$. For the dark matter halo we use the mass model of Sackett et al. (1994) with $\operatorname{disc} M / L=1$ in a spherical DM halo. From the circular velocity of the halo $v_{\mathrm{c}, \mathrm{h}}=160 \mathrm{~km} \mathrm{~s}^{-1}$ and with $2 \sigma_{\mathrm{h}}^{2}=v_{\mathrm{c}, \mathrm{h}}^{2}$ the halo velocity dispersion is $\sigma_{\mathrm{h}}=110 \mathrm{~km} \mathrm{~s}^{-1}$ and the halo density is $\rho_{\mathrm{h} 0}=v_{\mathrm{c}, \mathrm{h}}^{2} /\left(4 \pi G R_{0}^{2}\right)=4.7 \times 10^{-3} M_{\odot} \mathrm{pc}^{-3}$ leading to the surface density $\Sigma_{\mathrm{h}}=2 z_{\max } \rho_{\mathrm{h} 0}=47.5 M_{\odot} \mathrm{pc}^{-2}$ up to a maximum height of $z_{\max }=5.1 \mathrm{kpc}$. The initial values for the stellar disc are also taken from the total stellar mass in Sackett et al. (1994) and then iterated. The normalisation of the velocity dispersions are given by $\sigma_{\mathrm{e}}$ also determined iteratively from Eq. (18). 
The calculation of the self-consistent profile includes the intrinsic luminosity profiles in all bands using stellar population synthesis.

\subsection{Extinction and projection to the sky}

The next step is to choose the stellar scale height $z_{\mathrm{s}}$ (Eq. (18)), the radial scale lengths $R_{\mathrm{s}}$ and the cutoff radius $R_{\max }$ (Eq. (23)), the central surface density of stars $S_{0}$ (Eqs. (16), (17)) and the dust properties (Eq. (24)) including the inclination $i$ of the disc.

With these parameters the projection of the galaxy to the sky is calculated. We use a simple radiative transfer code neglecting scattered light. Some test runs performed by M. Xilouris has shown that scattered light contributes only a few percent to the surface brightness of typical edge-on galaxies. This is different to the significant influence of scattered light on the determination of the dust temperature in the disc (Misiriotis et al. 2001). With the $x$-axis along the line of sight, $y$-axis along the major axis, and $z$-axis along the minor axis we integrate for a set of vertical cuts highly resolved $z$-profiles of surface brightness profiles in $U, B, V, R$, and $I$. The resolution is $\mathrm{d} x \sin (i)=125 \mathrm{pc}$ along the line of sight and $\mathrm{d} z \sin (i)=12$ pc parallel to the minor axis and we restrict the integration to the cylinder bounded by $R_{\max }$ and $z_{\max }$. Possible foreground extinction due to dust outside $R_{\max }$ is neglected.

The extinction and reddening features of the vertical profiles are very sensitive to the dust geometry and the inclination (for a detailed discussion see Just et al. 1996). Therefore we test a large range of parameters to find the best match to the observed profiles.

Even if the match of the $V$-band profiles is not satisfactory we proceed to the multi-colour analysis to determine how to change the star formation history and/or the heating function.

\subsection{Multi-colour analysis}

Here we give an overview over the main effects on the vertical profiles due to the variation of the disc parameters. For details see Just et al. (1996). The global parameters of the stellar disc are determined by the surface brightness distribution at large heights $z$, where extinction can be neglected. The vertical cuts near the midplane are influenced by the dust distribution and the detailed distribution of the stellar subpopulations of different age.

We first discuss the influence of the dust distribution. If the dust scale height is larger than the scale height of the intrinsic star light near the midplane, then extinction leads to a flattening of the profile in the centre but no minimum (dust lane). Increasing the inclination results in a smearing out of the dust feature. Increasing the dust mass leads to a deeper and wider dust lane in the surface-brightness profiles but only a broadening in the colour index index profiles; also the radial extension becomes larger. With a radial scale length larger than the stellar scale length the extinction feature is distributed more shallowly in the radial direction and the dust lane shows a larger offset to the major axis.

The systematic variations of the shape of the vertical profiles from red to blue are not only a signature of reddening near the midplane, but are also due to the relative distribution of young and old stars. Changing the star formation history leads to a different fraction of luminous young blue subpopulations. A higher fraction of young and intermediate age populations with $\mathrm{O}, \mathrm{B}$ and A-stars gives an excess in the surface-brightness profiles
Table 1. The observational data of NGC 5907. Most columns are self explaining. FWHM is the full width half maximum of the seeing. The two last columns give the total magnitudes and errors from our measurements, corrected for galactic foreground extinction.

\begin{tabular}{cccccrc}
\hline \hline Filt & $\begin{array}{c}\text { Date } \\
\text { Jul97 }\end{array}$ & $\begin{array}{c}\text { Exp.time } \\
\text { [min] }\end{array}$ & Airmass & $\begin{array}{c}F W H M \\
\text { [arcsec] }\end{array}$ & $\begin{array}{r}\text { total } \\
\text { Magn }\end{array}$ & $\begin{array}{c}\text { phot } \\
\text { error }\end{array}$ \\
\hline$U$ & 11 & $8 \times 20$ & $1.10-1.70$ & 1.5 & 11.567 & \pm 0.070 \\
$B$ & 6 & $8 \times 20$ & $1.12-1.53$ & 1.5 & 11.279 & \pm 0.054 \\
$V$ & 5 & $4 \times 20$ & $1.06-1.13$ & 1.5 & 10.419 & \pm 0.057 \\
$R$ & 5 & $20+3 \times 10$ & $1.30-1.50$ & 1.3 & 9.701 & \pm 0.064 \\
$I$ & 5 & $4 \times 10$ & $1.17-1.26$ & 1.8 & 8.763 & \pm 0.084 \\
\hline
\end{tabular}

near the midplane which cannot be suppressed fully by dust extinction (especially in the red colours). The heating function determines the vertical spread of the subpopulations. Fast heating for the young populations results in centrally flattened surface brightness profiles combined with an extended blue regime.

As an example of the differences in the intrinsic luminosity and colour profiles we show in Fig. 12 a comparison model with constant SFR (large fraction of young stars) and the heating function of the solar neighbourhood (quick heating of young stars). The effect is a reduced central luminosity and a very extended blue regime. Therefore less dust is needed in the central region to obtain the observed surface brightness profiles. But the blue wings cannot be hidden by a reliable dust distribution.

\section{Observations and data reduction}

It is important to check the viability of our model by comparison with multi-colour observational data of NGC 5709. In this section we describe the observations. The comparison will be presented in Sect. 6 .

The observations were performed during July 5-11, 1997 using the $2.2 \mathrm{~m}$ telescope of the Calar Alto Observatory, Spain. The CAFOS focal reducer was used changing the focal ratio of the telescope from $\mathrm{f} / 8$ to $\mathrm{f} / 4.4$. The CCD camera was equipped with a SITE $2048 \times 2048$ pixel chip. The pixel size was $24 \mu$, corresponding to 0.53 arcsec. The circular field-of-use had a diameter of 16 arcmin. Standard Johnson $U, B, V$ and Cousins $R, I$ filters were used. Several images were exposed in each filter without interrupting the tracking of the telescope. The exposure times in UBVRI are listed in Table 1.

Sky flats were exposed in every dawn and dusk phase. The photometric quality was good or very good in all nights. Photometric standard stars $(\approx 40)$ in the globular cluster M 92 were exposed every night (Christian et al. 1985; Sandage \& Walker 1966; Cathey 1974; Stetson \& Harris 1988).

\subsection{Data reduction}

The MIDAS program system was used for the data reduction. From comparison and evaluation of all bias exposures an optimal working bias image was constructed. Similarly, from comparison and evaluation of all flat-field exposures optimal workingflat-field images were constructed for each colour. Bias subtraction and flat-field division were performed in the usual manner for each image. The flatness of the sky was checked in each image and corrected interactively, if necessary. The flatness of the sky down to less than $0.5 \%$ is an important condition for a reliable model of the surface-brightness distribution. 


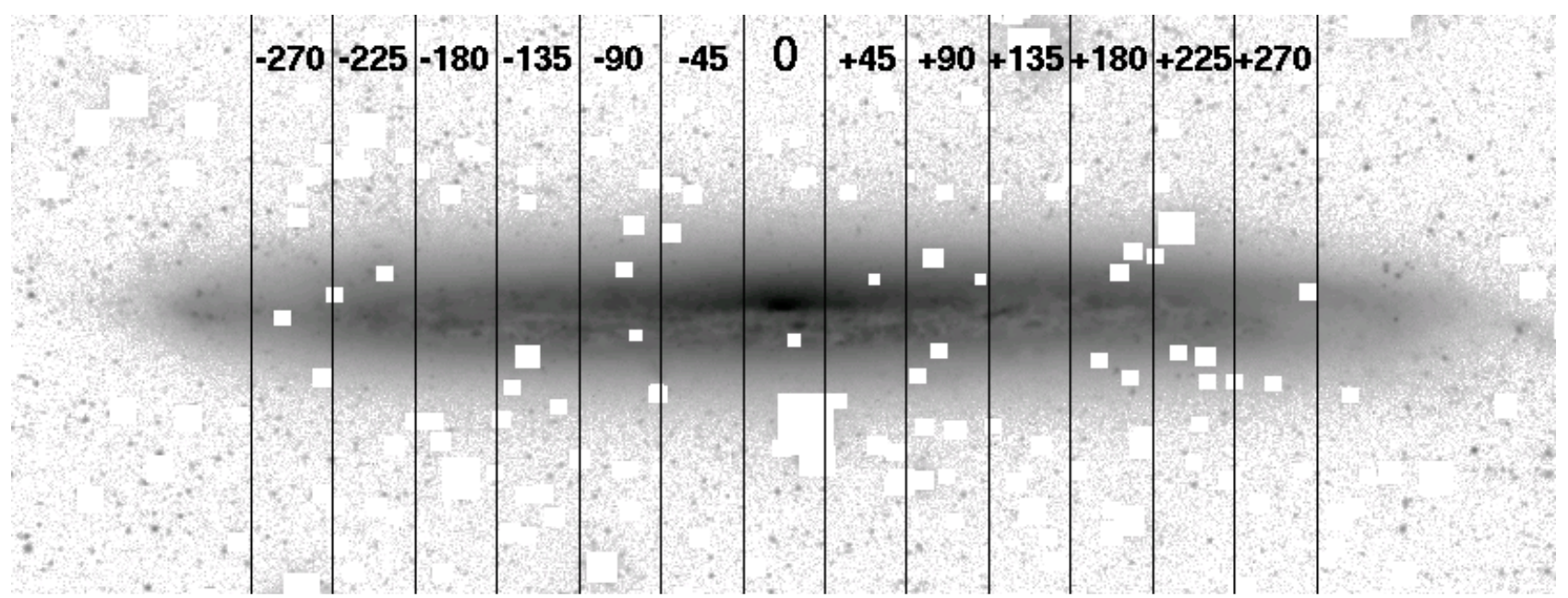

Fig. 6. Deep $V$ image of NGC 5907 with the stellar mask. The image is rotated for a horizontal orientation of the galaxy. The field of view is $850 \times 320$ arcsec. The vertical flux profiles were calculated by mean values over the 13 indicated stripes of 45 arcsec width. The black lines mark the borders of these stripes. The flux values for the each point of the profiles were calculated by averaging along horizontal pixel rows of 45 arcsec length. The numbers in the stripes give their corresponding central positions in arcsec. NW is left, SE is right.

The images in each colour were added together to a total image. A rebinning was not necessary since the tracking of the telescope was not interrupted between the exposures (this was checked, cf the column FWHM in Table 1). By stacking the images in the vertical direction it was possible to remove the cosmic pixels during the summation (Kappa-sigma-summation). Figure 1 shows as an example the total image in $R$. The faint filament extending northwards from the left part of the disk is real. It probably belongs to a remnant of an interacting dwarf galaxy (Shang et al. 1996).

The flux calibration was calculated using the standard stars. The zero-points could be determined with a error between 0.05 (B) to $0.08(I) \mathrm{mag}$. The atmospheric extinction of the corresponding airmass was calculated for each single exposure. Each exposure was considered with a corresponding weight in the flux calibration of the total image. The galactic foreground extinction for NGC 5907 is rather small: $A_{\mathrm{g}}(\mathrm{B})=0.01$ taken from RC3 (de Vaucouleurs et al. 1991), see also in Burstein \& Heiles (1984). The extinction corrections for the other filters were computed by the coefficients given in Cardelli et al. (1989) (their Table 3). The resulting corrections were $A_{\mathrm{g}}=$ $0.012,0.010,0.008,0.006,0.004 \mathrm{mag}$ for $U, B, V, R, I$, respectively. Because of the small distance of NGC 5907 the $\mathrm{K}$-corrections are very small and were not considered.

For the total magnitude of NGC 5907 we obtain the values given in Table 1 . These values are corrected for galactic foreground extinction. The errors are between 0.05 and 0.08 mag. The corresponding $B$ value from the RC3 is $B_{\text {tot }}=11.11 \pm 0.10$. Our value is 0.17 mag weaker. The difference might be explained by the fact that the RC3 value was obtained by aperture photometry while we have masked the foreground stars in front of and around the galaxy.

\subsection{Surface-brightness and colour index profiles}

For the comparison with the models we need vertical profiles of the calibrated flux in $U B V R I$ and the corresponding colour indices. All arithmetic calculations for this were done with the flux images. The flux calibrations were done afterwards.

The total images were rotated to set the galaxy into a horizontal position. A mask with appropriate squares around all fairly bright foreground stars and background galaxies was constructed from the rotated total $V$ image.

For the construction of vertical flux profiles a number of corresponding vertical stripes were defined. The centre of the first stripe was placed at the centre of the galaxy and the others were placed at the horizontal positions \pm 45 , $\pm 90, \pm 135, \pm 180, \pm 225, \pm 270$ arcsec along the disk (Fig. 6). This corresponds to $0.5,1.0,1.5,2.0,2.5$, and 3.0 scale lengths. Each stripe had a width of 45 arcsec, thus the whole disk was covered. In each stripe we calculated the mean flux along each horizontal pixel row of 45 arcsec length. Whenever a stellar mask was hit, this was adequately considered in the mean value. In this way we obtained 13 flux profiles over $z$ for each colour $U, B, V, R, I$. Further on we calculated the average of the left and right profiles of the corresponding horizontal positions.

Using our flux-calibration formula these profile were transformed into magnitudes. The profiles of the colour indices $U-B$, $B-V, V-R, R-I$ were obtained by subtraction of the corresponding profiles.

In Fig. 7 we show the vertical cuts in $U$ - and $V$-band as examples. The thin lines are the NW- and SE-profile and the thick lines are the averaged profiles which we used for the model. The extinction feature (perturbed by the clumpy structure of the young subpopulation) is clearly visible. In the outer parts the profiles are exponential and the noise level is well defined. The small offsets of the profiles in the different profiles show that the warp discussed in Morrison et al. (1994) seems to be not very systematic or is only significant for the young clumpy disc component.

\section{Results}

We show the comparison of the final model to the data in the surface-brightness and colour index profiles. Then we give the global parameters of the disc model, describe the scaling procedure, and discuss the intrinsic properties of the model.

\subsection{Comparison with the data}

In Fig. 9 the observed and model vertical surface-brightness profiles in all five bands are shown. The five profiles in each plot 

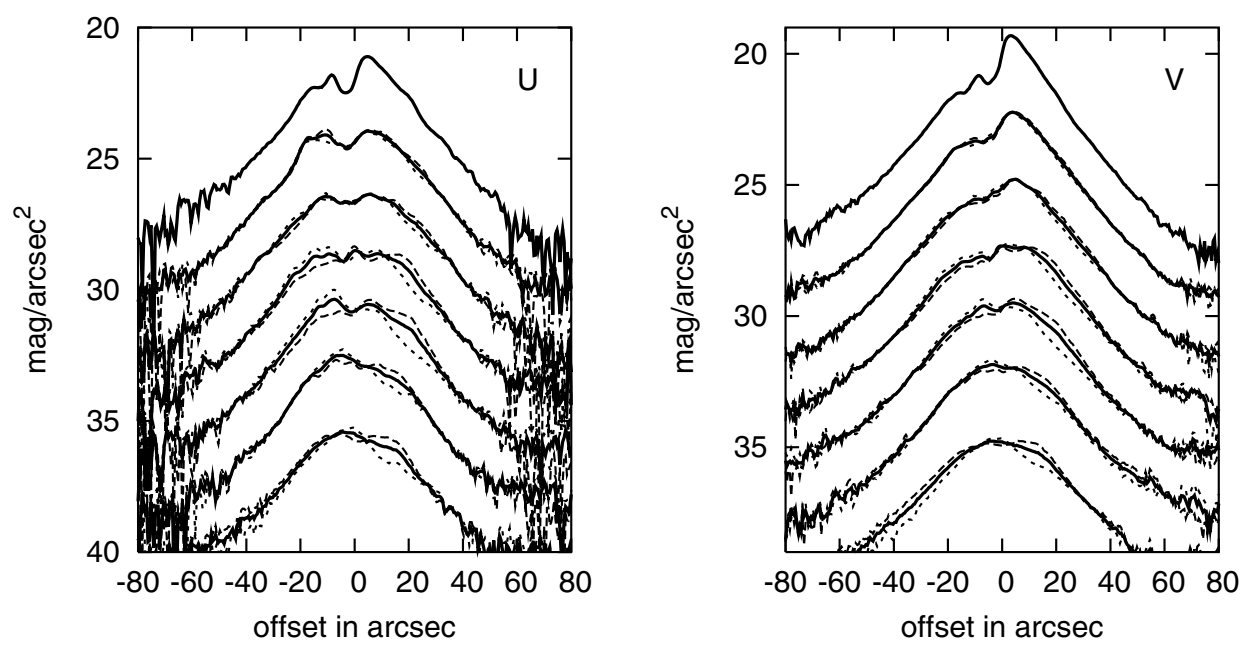

Fig. 7. Vertical surface-brightness profiles of NGC 5907 each averaged over $45^{\prime \prime}$ in the radial direction with an offset to the minor axis of 0 , 45, $90,135,180,225$, and $270^{\prime \prime}$ from top to bottom. For a better visibility the magnitude scale is shifted by 2 magnitudes from one to the next. Long and short dashed lines give the individual profiles for the $\mathrm{NW}(-)$ and $\mathrm{SE}(+)$ sides of the disc and the thick full lines are the averaged profiles.
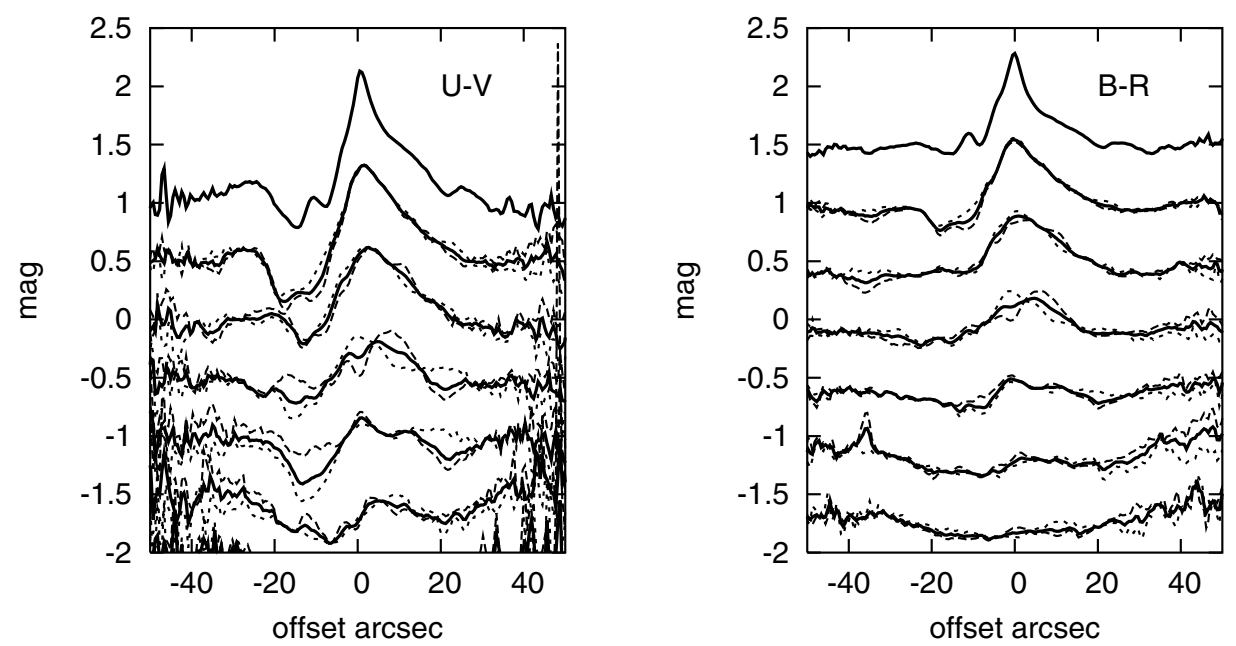

Fig. 8. Vertical profiles of the colour indices $U-V$ and $B-R$ for NGC 5907 for the same cuts as in Fig. 7. From top to bottom, the magnitude scale is shifted in colour by $-0.5 \mathrm{mag}$ from one to the next.

are vertical cuts parallel to the minor axis at distances along the major axes of $0,2.4,4.8,7.2,9.6,12.0$, and $14,4 \mathrm{kpc}$ from top to bottom, respectively. The general features in the discdominated parts are reproduced reasonably well by the model in all bands. In the inner two profiles the additional bulge light can be observed and far above the galactic plane an additional more extended component appears as discussed in Lequeux et al. (1996). Only the $U$-band profiles show a systematic deviation in the sense that they seem to be radially more extended. The extinction features near the midplane are of a similar strength as observed but due to the strong clumping not reproduced in detail. The large optical depth totally obscures the strong excess stellar light in the intrinsic profiles (see Fig. 12).

The corresponding colour index profiles (Fig. 10) are relatively flat as expected even at high optical depth due to the mixture of stellar emissivity and dust extinction along the line of sight (see Just et al. 1996). The systematic shape variation of the reddening features in the colour indices $U-B, B-V, V-R$, and $R-I$ running from asymmetric with blue dip in the blue colours to more symmetric and redder in the red colours is also reproduced.

\subsection{Global properties}

The disc parameters of the final model are collected in Table 2. The inclination $i=87.5^{\circ}$ and the cutoff radius $R_{\max }=19.7 \mathrm{kpc}$ are very similar to the model of Xilouris et al. (1999). The radial and vertical scale length $R_{\mathrm{s}}=5.5 \mathrm{kpc}$ and $z_{\mathrm{s}}=520 \mathrm{pc}$ are somewhat larger, because we concentrate our fit on the height regime $|z|<1.5 \mathrm{kpc}$, where the Xilouris et al. fit is not satisfactory.

For the total stellar mass (luminous plus remnants) we obtain from the central surface density $\Sigma_{\mathrm{s}, 0}=115 M_{\odot} \mathrm{pc}^{-2}$ a total stellar mass of

$M_{\mathrm{S}}=1.9 \times 10^{10} M_{\odot}$

corresponding to an average star formation rate of $\langle S F R\rangle=$ $M_{\mathrm{s}} / g_{\mathrm{eff}} / t_{\mathrm{a}}=2.34 M_{\odot} / \mathrm{yr}$ and a present day value of $S F R_{0}=$ $1.64 M_{\odot} / \mathrm{yr}$.

The dust component has a radial and vertical scale length of $R_{\mathrm{d}}=7.7 \mathrm{kpc}$ and $z_{\mathrm{d}}=110 \mathrm{pc}$, respectively. The central extinction coefficient is $A_{\mathrm{V}, 0}=4.0 \mathrm{mag} / \mathrm{kpc}$, which corresponds to a face-on optical depth of $\tau_{\mathrm{V}}^{\mathrm{f}}=0.81$ and central gas density of $\rho_{\mathrm{d}, 0}=7.0 \times 10^{-4} M_{\odot} / \mathrm{pc}^{3}$. The total dust mass is 

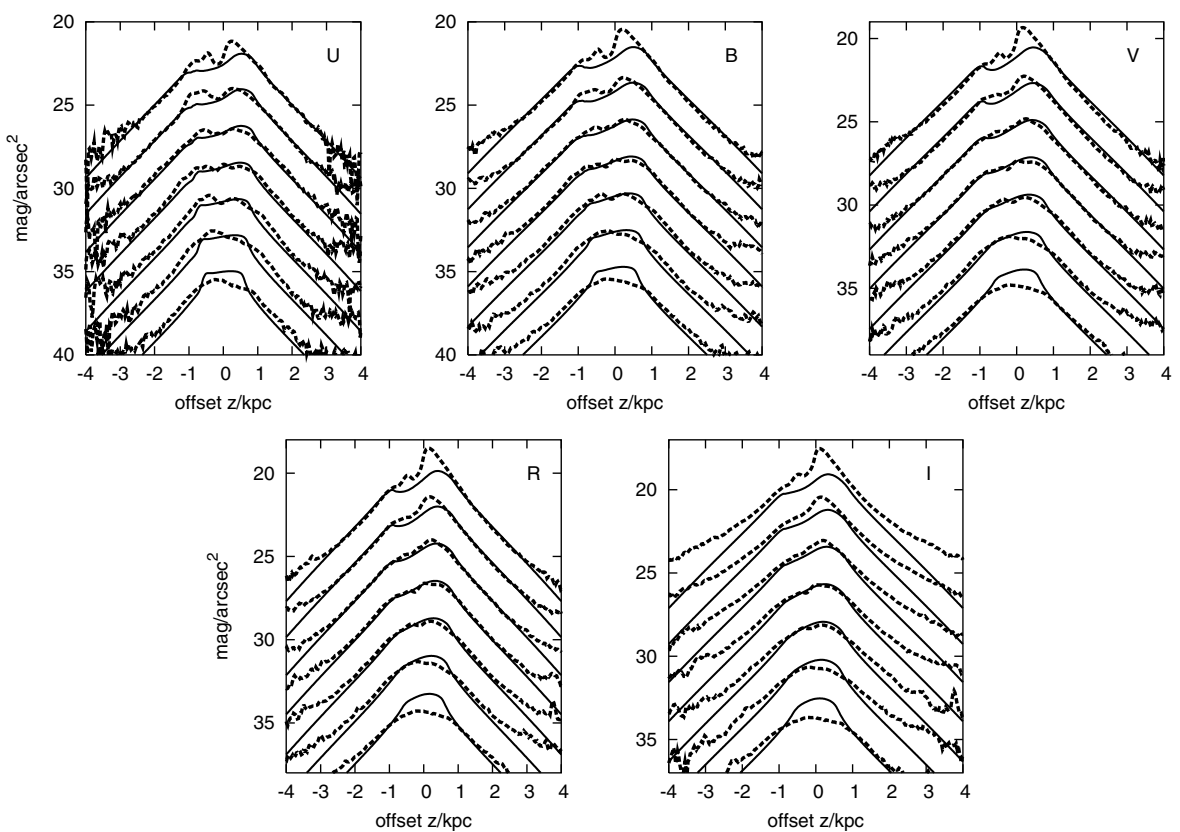

Fig. 9. Comparison between observations and model. Vertical surface brightness profiles for NGC 5907 in the $U, B, V, R$, and $I$ bands from upper left to lower right. The seven profiles in each plot are vertical cuts at distances along the major axes of $0,45,90,135,180,225$, and $270^{\prime \prime}$ corresponding to $0,2.4,4.8,7.2,9.6,12.0$, and $14.4 \mathrm{kpc}$ from top to bottom. Dashed lines represent the data, while solid lines give the model. From top to bottom, the magnitude scale is shifted downwards in brightness by $0,2,4,6,8,10$, and 12 magnitudes, respectively.
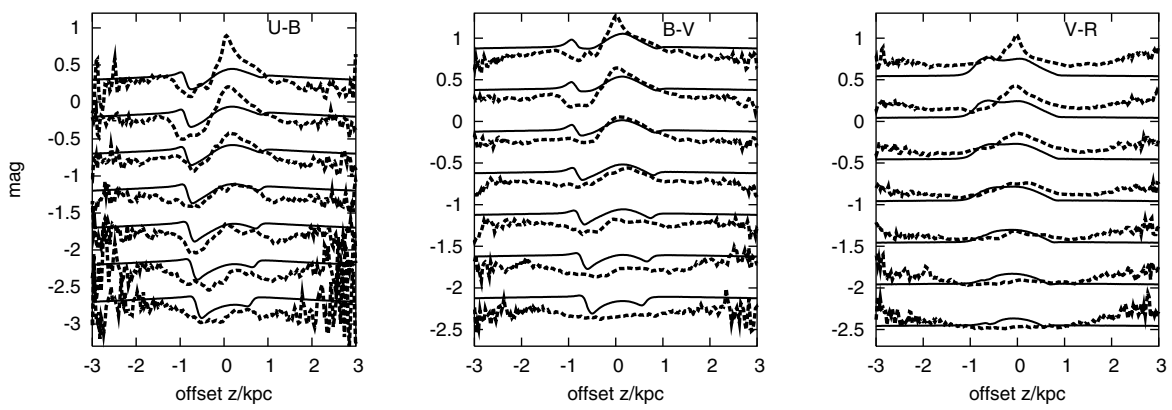

Fig. 10. Vertical profiles for NGC 5907 in the $U-B, B-V, V-R$, and $R-I$ colour indices from upper left to lower right. The seven profiles in each plot are the same vertical cuts as in Fig. 9. Dashed lines represent the data, while solid lines give the model. From top to bottom, the magnitude scale is shifted by $0,1,2,3,4,5$ and 6 magnitudes to the blue, respectively.

Table 2. Parameters of the final model for NGC 5907. At the left hand side the physical quantities of the stellar disc are given and at the right hand side the parameters for the dust component. Inclination $i$ and cutoff radius $R_{\max }$ are the same for all components. $S_{0}$ is the integrated star formation at the centre and $g_{\text {eff }}$ is the conversion factor to the stellar surface density $\Sigma_{\mathrm{s}, 0}=g_{\mathrm{eff}} S_{0}=115 M_{\odot} \mathrm{pc}^{-2} . C_{z}$ is the shape correction factor of the stellar density profile.

\begin{tabular}{lrlr}
\hline \hline \multicolumn{2}{c}{ stellar disc } & \multicolumn{2}{c}{ dust component } \\
\hline$i$ & $87.5^{\circ}$ & & \\
$R_{\max }[\mathrm{kpc}]$ & 19.7 & & 7.7 \\
$R_{\mathrm{S}}[\mathrm{kpc}]$ & 5.5 & $R_{\mathrm{d}}[\mathrm{kpc}]$ & 110 \\
$z_{\mathrm{S}}[\mathrm{pc}]$ & 520 & $z_{\mathrm{d}}[\mathrm{pc}]$ & 4.0 \\
$S_{0}\left[M_{\odot} \mathrm{pc}^{-2}\right]$ & 170 & $A_{\mathrm{V}, 0}[\mathrm{mag} / \mathrm{kpc}]$ & \\
$g_{\mathrm{eff}}$ & 0.676 & & \\
$C_{z}$ & 1.05 & & \\
\hline
\end{tabular}

$M_{\mathrm{d}}=4 \pi R_{\mathrm{d}}^{2} z_{\mathrm{d}} \rho_{\mathrm{d}, 0}=5.7 \times 10^{7} M_{\odot}$ leading to a gas-to-dust ratio of $F=M_{\mathrm{g}} / M_{\mathrm{d}}=209$.

\subsection{The scaling radius $R_{0}$}

The input parameters of gas and halo component for the intrinsic disc structure are determined at the scaling radius $R_{0}=10 \mathrm{kpc}$ (see Table 3). For the normalisation we need the properties of the stellar component from the final model. The stellar surface density is $\Sigma_{\mathrm{s}}=18.7 M_{\odot} \mathrm{pc}^{-2}$ (Eq. (23)) and the maximum velocity dispersion is $\sigma_{\mathrm{e}}=32 \mathrm{~km} \mathrm{~s}^{-1}$ (Eq. (18)).

For the gas component we adopt the same scale lengths as for the dust, i.e. $R_{\mathrm{g}}=R_{\mathrm{d}}=7.7 \mathrm{kpc}$ and $z_{\mathrm{g}}=z_{\mathrm{d}}=110 \mathrm{pc}$, which for the radial distribution is consistent with the larger extension of the HI distribution. The vertical scale height corresponds to the maximum age bin of $t_{\mathrm{g}}=3 \mathrm{Gyr}$. This can be converted to a maximum velocity dispersion of $\sigma_{\mathrm{g}, \mathrm{m}}=0.325 \sigma_{\mathrm{e}}=10.4 \mathrm{~km} \mathrm{~s}^{-1}$ and an rms value of $\sigma_{\mathrm{g}}=0.16 \sigma_{\mathrm{e}}=5.1 \mathrm{~km} \mathrm{~s}^{-1}$ for the gas.

For the DM halo the surface density and the velocity dispersion taken from the mass model of Sackett et al. (1994) $\Sigma_{\mathrm{h}}=48.5 M_{\odot} \mathrm{pc}^{-2}$ up to a maximum height of $z_{\max }=5.1 \mathrm{kpc}$ and $\sigma_{\mathrm{h}}=110 \mathrm{~km} \mathrm{~s}^{-1}$ (see Sect. 4.1) are used. Since we deduced the halo properties from the rotation curve, we checked the reconstruction of the rotation curve with our final model. 
Table 3. Input parameters for the final model of NGC 5907. At the left hand side the physical quantities at $R_{0}=10 \mathrm{kpc}$ are given and at the right hand side the corresponding normalised parameters of the normalised profile. The corresponding description is given in Sect. 6.3.

\begin{tabular}{lrlr}
\hline \hline \multicolumn{2}{c}{ at $R_{0}=10 \mathrm{kpc}$} & \multicolumn{2}{c}{ scaling parameter } \\
\hline$z_{\mathrm{s}}[\mathrm{pc}]$ & 520 & $\sigma_{\mathrm{e}}\left[\mathrm{km} \mathrm{s}^{-1}\right]$ & 32 \\
$\Sigma_{\mathrm{s}}\left[M_{\odot} \mathrm{pc}^{-2}\right]$ & 18.7 & $Q_{\mathrm{s}}$ & 0.25 \\
$\Sigma_{\mathrm{g}}$ & 8.8 & $Q_{\mathrm{g}}$ & 0.11 \\
$\Sigma_{\mathrm{h}}$ & 48.5 & $Q_{\mathrm{h}}$ & 0.64 \\
$\sigma_{\mathrm{h}}\left[\mathrm{km} \mathrm{s}^{-1}\right]$ & 110 & $s_{\mathrm{h}}$ & 3.5 \\
$z_{\mathrm{g}}[\mathrm{pc}]$ & 110 & $t_{\mathrm{g}}[\mathrm{Gyr}]$ & 3.0 \\
\hline
\end{tabular}

A mass model leading to an equally good fit of the rotation curve in Sackett et al. can be constructed by a four component galaxy including a Hernquist bulge, exponential discs for the stellar and the gas component, and a pseudo-isothermal dark matter halo. The bulge with a mass of $3 \times 10^{10} M_{\odot}$ and a core radius of $1 \mathrm{kpc}$ reproduces the steep rise of the $\mathrm{CO}$ rotation curve presented in Sofue et al. (1996). We adopt a flattening of 0.5 as determined in Barneby \& Thronson (1994). The exponential discs are determined by the scalelengths of $5.5 \mathrm{kpc}$ and $7.7 \mathrm{kpc}$ and the total masses of $1.9 \times 10^{10} M_{\odot}$ and $1.2 \times 10^{10} M_{\odot}$ (corresponding to $2.18 \times 10^{10} M_{\odot}$ and $1.65 \times 10^{10} M_{\odot}$ when neglecting the cutoff) for stars and gas, respectively. The dark matter halo has a local density of $5.5 \times 10^{-3} M_{\odot} / \mathrm{pc}^{3}$ at $R_{0}=10 \mathrm{kpc}$ with a core radius of $1 \mathrm{kpc}$. The local density is slightly higher than that used in our model in order to correct for the effect of the core. The rotation curve of NGC 5907 presented in Sofue et al. (1996) is significantly different and also shows the uncertainties in the observational data itself. To reproduce the prominent maximum around a radius of $12 \mathrm{kpc}$, one can use a NFW halo with scale radius of $6 \mathrm{kpc}$ and local density of $4.78 \times 10^{-3} M_{\odot} / \mathrm{pc}^{3}$ (as in our model) combined with a smaller bulge with core radius $0.5 \mathrm{kpc}$ and mass of $1.2 \times 10^{10} M_{\odot}$. There is a large freedom in the parameter choice to reproduce the rotation curves, especially if we allow the disk mass to vary. Instead of using a NFW profile the maximum in the rotation curve of Sofue et al. can also be reached by increasing the disc mass by a factor of 2-3.

The total surface density at $R_{0}$ is $\Sigma_{\mathrm{tot}}=76.0 M_{\odot} \mathrm{pc}^{-2}$, which is used to determine the ratios $Q_{\mathrm{s}}=\Sigma_{\mathrm{s}} / \Sigma_{\text {tot }}$ etc. The halo velocity dispersion is normalised by $s_{\mathrm{h}}=\sigma_{\mathrm{h}} / \sigma_{\mathrm{e}}=3.5$. All input parameters of the final model for NGC 5907 are collected in Table 3.

\subsection{Intrinsic disc properties}

The self-consistent vertical profiles of the final model are shown in Fig. 11. The stellar density profile is approximately exponential with an excess below $|z| \approx 500 \mathrm{pc}$. At the midplane the stellar density compared to the extrapolated exponential profile shows an excess of a factor of 2.1. The corresponding excess in surface density is $48 \%$. The gas density profile matches the scale height of the dust component with $z_{\mathrm{g}}=z_{\mathrm{d}}=110 \mathrm{pc}$. The central gas density is slightly (5\%) larger than the central stellar density. The DM halo profile is very flat and decreases by $44 \%$ from the midplane to $z_{\max }=5.1 \mathrm{kpc}$. The halo density exceeds the disc density (stars plus gas) for $|z|>510 \mathrm{pc}$, but the cumulative mass, which determines the local gravitational force, dominates only at $|z|>2.7 \mathrm{kpc}$.

The left panels in Fig. 12 show the intrinsic luminosity profiles in all bands. For comparison the density profile is also plotted to emphasise the enhanced stellar light of the young stars near the midplane. As the lower panel shows for the $B$-band,

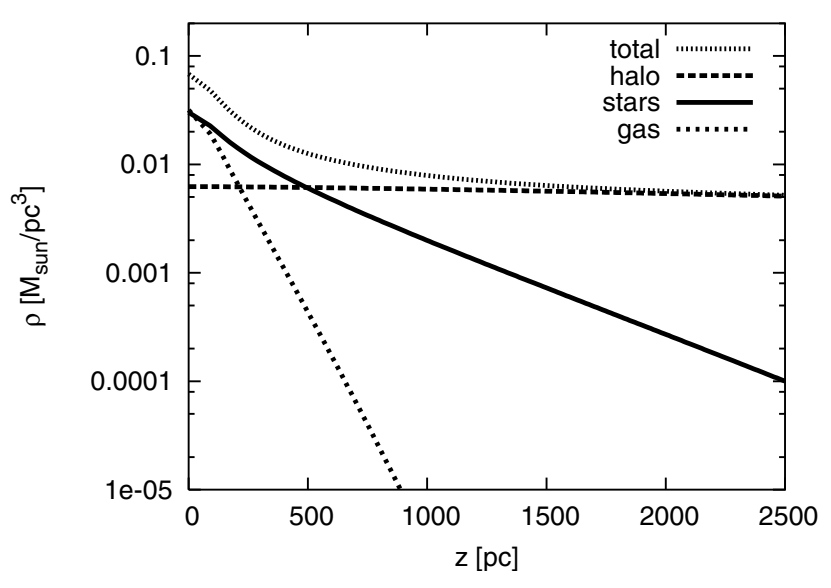

Fig. 11. Vertical density profiles of the final of NGC 5907 model normalised to the values at the scaling radius $R_{0}=10 \mathrm{kpc}$. The gas density (short dashed line) slightly dominates towards the midplane. The stellar density (full line) has an exponential scale height of $z_{\mathrm{s}}=520 \mathrm{pc}$ with a density enhancement to the midplane. The halo density (long dashed line) shows only $20 \%$ variation below $2.5 \mathrm{kpc}$ due to the adiabatic contraction.

Table 4. Intrinsic properties of the final model of NGC 5907 in the different bands. The absolute quantities (central emissivity, surface brightness and total luminosity) depend on the central stellar surface density and the radial and vertical scale length as given in Table 2. The first row gives the central emissivity $\epsilon_{0}$ and the second row shows the enhancement factor $\left(\epsilon / \epsilon_{\exp }\right)_{0}$ with respect to the exponential extrapolation form $z \approx 3 z_{\mathrm{s}}$ to the midplane. The mass-to-light ratios $(M / L)_{0}$ at the midplane are very low due to the high fraction young stars. The next two rows give the central face-on surface brightness $L(R=0)$ in physical and observational units. $m_{\text {tot }}$ is the total apparent luminosity with the distance module $\Delta m=30.2 \mathrm{mag}$ and $\Delta m_{\mathrm{te}}=m_{\mathrm{tot}}-m_{\exp }$ quantifies the additional stellar light compared to pure exponential vertical profiles. The average stellar mass-to-light ratios $(M / L)_{\text {tot }}$ of the model are the same for each vertical profile and the whole disc. For the exponential extrapolation $(M / L)_{\text {exp }}$ is constant all over the disc and equals the $M / L$ of the model at large heights $z$.

\begin{tabular}{clccccc}
\hline \hline & unit & $U$ & $B$ & $V$ & $R$ & $I$ \\
\hline$\epsilon_{0}$ & $\mathrm{mag} / \mathrm{pc}^{3}$ & 6.05 & 6.17 & 5.81 & 5.50 & 5.10 \\
$\left(\epsilon / \epsilon_{\text {exp }}\right)_{0}$ & - & 23.3 & 15.4 & 9.46 & 7.59 & 6.31 \\
$(M / L)_{0}$ & $(M / L)_{\odot}$ & 0.27 & 0.35 & 0.45 & 0.56 & 0.53 \\
\hline$L_{0}$ & $\mathrm{mag} / \mathrm{pc}^{2}$ & 0.15 & 0.09 & -0.50 & -0.93 & -1.43 \\
& $\mathrm{mag} / \prime^{\prime 2}$ & 22.73 & 22.66 & 21.05 & 20.64 & 20.14 \\
\hline$m_{\text {tot }}$ & $\mathrm{mag}$ & 9.65 & 9.59 & 9.0 & 8.57 & 8.07 \\
$\Delta m_{\mathrm{te}}$ & $\mathrm{mag}$ & -1.78 & -1.51 & -1.21 & -1.09 & -0.99 \\
$(M / L)_{\mathrm{tot}}$ & $(M / L)_{\odot}$ & 0.80 & 0.88 & 0.91 & 1.00 & 0.87 \\
$(M / L)_{\exp }$ & $(M / L)_{\odot}$ & 2.78 & 2.39 & 1.88 & 1.85 & 1.46 \\
\hline
\end{tabular}

the profiles deviate from the exponential shape already at heights below $|z|=1 \mathrm{kpc}$. In the right panels the corresponding colour profiles and mass-to-light ratios are shown. The intrinsic colours above $|z| \approx 200$ pc show very shallow gradients for this model. The colour gradient due to the blue young population can be seen only in the innermost part. The mass-to-light ratio is more sensitive to the age-distribution of the stars and varies much more strongly than the colours. It varies by a factor of 10 in $U$-band and even in $I$-band by a factor of 3 . The $M / L$-gradient is significantly larger than zero up to $|z| \approx 500 \mathrm{pc}$ at least in the blue bands. The average value of $M / L$ corresponds to the local value near a height of $|z| \approx 150 \mathrm{pc}$ (dots in the lower right panel of Fig. 12). 

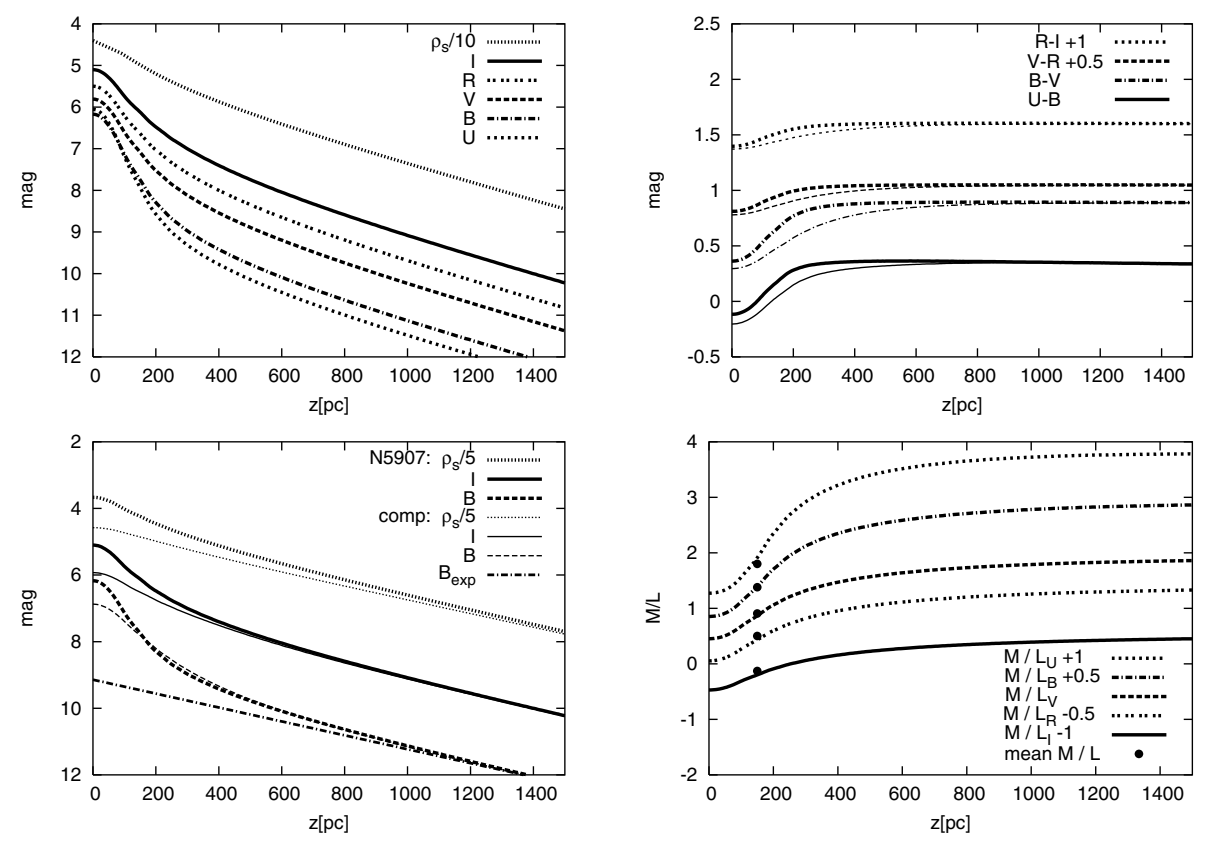

Fig. 12. Upper left panel: the emissivity of the central profile of NGC 5907 in mag pc ${ }^{-3}$. For comparison the stellar density profile is also plotted in units of $M_{\odot} \mathrm{pc}^{-3} / 10$ (also in mag). Lower left panel: the $B$ and $I$-band emissivity for comparison with a model using the heating function of the solar neighbourhood and a constant star formation rate. For the $B$-band profile the exponential profile fit (dot-dashed line) is shown. The corresponding density profiles are normalised to $M_{\odot} \mathrm{pc}^{-3} / 5$. Upper right panel: intrinsic colour profiles at the centre of NGC 5907 (thick lines). The profiles are shifted to avoid overlap. The profiles of the comparison model (thin lines) with constant $S F R$ show more extended colour gradients due to the faster dynamical heating (see Fig. 2). Lower right panel: the mass-to-light ratios in all bands, also shifted to avoid overlap. The dots denote the average values for the total surface density (see Table 4).

The luminosity weighted mean metalicity of the final model varies from $[\mathrm{Fe} / \mathrm{H}]=0.06$ at the midplane to $[\mathrm{Fe} / \mathrm{H}]=-0.59$ at high latitude $|z|$, which is only slightly less than the variation in the chemical enrichment of the subpopulations.

In order to show that the evolutionary history cannot be decomposed arbitrarily into a dynamical heating function and an appropriate star formation history, we calculated comparison models, where we have fixed the heating function by that of the solar neighbourhood (Fig. 2 lower panel, thin line) and changed the $S F R$ in order to get a higher fraction of young stars. But even with a constant $S F R$ the strong heating of the young subpopulations spread the blue stars to a much larger height yielding more extended colour gradients and a smaller stellar light excess near the midplane (thin lines in Fig. 12).

\section{Discussion}

\subsection{Disc evolution}

Our final model combines a star formation history with a moderate maximum at an age of $t=7.2 \mathrm{Gyr}$ with a slow dynamical heating for the young stellar population compared to the solar neighbourhood (see Fig. 2). If we use as in the comparison model the heating function of the solar neighbourhood (constant increase of kinetic energy of the subpopulations with age), a higher present day $S F R$ is necessary to get a comparable luminosity in the blue bands near the midplane. Even a constant $S F R$ (which is often claimed even for the Milky Way) does not reach the intrinsic luminosity of our final model, but leads to additional blue light at intermediate heights $|z|$, where dust reddening cannot compensate. We do not claim that the chosen $S F R$ is unique, because the parameter space is too large and a model on a much higher level of comparison including radial variation of the intrinsic profiles and clumping near the midplane would be necessary. Our result shows that a continously decreasing $S F R$ in the last few Gyr can account for the observed high intrinsic luminosity at the midplane necessary for the strong NIR-submm brightness. A recent star burst is not needed but also not excluded by our model at this level of modelling using a simple radial disc structure.

\subsection{Mass-to-light ratio}

The stellar mass-to-light ratio $M / L$ strongly depends on the $I M F$ at the low mass end without changing the luminosities much. In our model with the Scalo-IMF we get a relatively low value $M / L_{\mathrm{V}}=0.91$, which fits well to the mass models of Sackett et al. (1994). Since the disc of an Sc galaxy is younger than an Sbc galaxy like the Milky Way, this value also fits well compared to the values in the solar neighbourhood. A rough estimate of the excess in blue light of the young stellar population in our model compares well to the additional light needed in Misiriotis et al. (2001) to heat the dust. Their corresponding mass-to-light ratio and recent $S F R$ are slightly different, because they used a different stellar population model (Salpeter IMF and no mass loss due to stellar evolution).

The low mass-to-light ratio of the stellar disc is a clear indication of a low disc mass model as discussed also in Sackett et al. (1994). Our underlying mass model is consistent with the observed rotation curve of Sackett et al. with a pseudo-isothermal DM halo and for the rotation curve of Sofue et al. (1996) a NFW halo fits better. But since the mass model is fully self-consistent at the scale radius $R_{0}=10 \mathrm{kpc}$ only, we cannot distinguish between isothermal or NWF profiles for the dark matter halo. 


\subsection{Dust component}

For the dust component we find a similar scale height as Misiriotis et al. (2001) and a similar total dust mass, which is responsible for the FIR brightness. A significant difference to the models of Xilouris et al. (1999) and Misiriotis et al. is the larger dust scale length of $R_{\mathrm{d}}=7.7 \mathrm{kpc}$. The effect of a larger $R_{\mathrm{d}}$ is, apart from the stronger extinction feature at large distance form the minor axis, a shift of the extinction dip to higher vertical offset at all vertical cuts. This effect cannot be substituted by a larger inclination $i$, because this would smear out the extinction feature to larger heights above the major axis. A side effect of the larger radial scale length is the reduced face-on optical depth at the centre $\left(\tau_{\mathrm{V}}^{\mathrm{f}}=0.81\right.$ instead of 1.5). Xilouris et al. also found that the dust scale length is larger than the stellar scale length in their best fit. The large radial scale length of the dust also fits to the more extended distribution of HI and of the FIR brightness.

\section{Conclusions}

We have observed deep photometry of NGC 5907 in the $U, B, V$, $R$, and $I$-band in order to construct a physical disc model based on a multi-colour analysis. We have constructed a self-consistent evolutionary disc model for NGC 5907, which fits the general characteristics of the surface brightness distribution in all bands. The stellar disc is built up by a sequence of stellar subpopulations distributed according to a smooth SFR and dynamical heating function $\sigma(t)$. The emissivity is calculated with stellar population synthesis and the appearance of the galaxy is calculated by radiative transfer including an exponential disc component of dust.

This is the first physical model of the disc of NGC 5907 that gives the correct luminosities and colours of the outer exponential parts of the disc and also the large amount of dust observed in FIR/submm. The reason for the corresponding excess in stellar light near the midplane of the disc is the concentrated luminous young subpopulation produced by the continuous SFR. Our model is a significant improvement of the disc model of Misiriotis et al. (2001), who combined the phenomenological exponential model of Xilouris et al. (1999) with an additional thin disc of uniformly mixed young stars and dust in order to produce the excess in FIR/submm luminosity.

This evolutionary disc model naturally explains three different aspects known from the literature: 1) The exponential vertical structure well above the midplane as modelled phenomenologically by Xilouris et al. (1999) is the result of the older subpopulations in dynamical equilibrium in the total disc potential. 2) The large amount of dust observed in the FIR/submm (modelled by Misiriotis et al. 2001) is necessary to hide the bright young subpopulations confined to the midplane. The young stars are the smooth end of the $S F R$ without a recent star burst. 3) The additional light necessary to heat the dust is automatically delivered by the high mass-to-light ratio near the midplane.

Acknowledgements. We thank the Deutsche Forschungsgemeinschaft for supporting this project by the SFB 328 and SFB 439 at the University of Heidelberg. We are grateful to the staff of the Calar Alto Observatory for the support during the observations.

\section{References}

Barneby, D., \& Thronson, Jr. H. A. 1994, AJ, 107, 1717

Binney, J., \& Merrifield, M. 1998, Galactic Dynamics, Princeton Univ. Press, 137

Burstein, D., \& Heiles, C. 1984, ApJS, 54, 33

Cardelli, J. A., Clayton, G. C., \& Mathis, J. S. 1989, ApJ, 345, 245

Cathey, L. R. 1974, AJ, 79, 1370

Christian, C. A., Adams, M., Barnes, J. V., et al. 1985, PASP, 97, 363

de Grijs, R., \& Peletier, R. F. 2000, MNRAS, 313, 800

de Vaucouleurs, G., de Vaucouleurs, A., Corvin, H. G. Jr., et al. 1991, Third Reference Catalogue of Bright Galaxies (New York: Springer)

Draine, B. T., \& Lee, H. M. 1984, ApJ, 285, 89

Dumke, M., Braine, J., Krause, M., et al. 1997, A\&A, 325, 124

Edvardsson, E., Andersen, J., Gustafsson, B., et al. 1993, A\&A, 275, 101

Fioc, M., \& Rocca-Volmerange, B. 1997, A\&A, 326, 950

Freeman, K. C. 1991, in Sundelius B., Dynamics of Disc Galaxies. Göteborg Observatory, Göteborg, 15

Guthrie, B. N. G. 1992, A\&A Supp. Ser., 93, 255

Jahreiss, H., \& Wielen, R. 1997, in Proc. ESA SP-402, HIPPARCOS '97, ed. B. Battrick, M. A. C. Perryman, \& P. L. Bernacca (Nordwijk, ESA), 675

Just, A. 2001, Dynamics of Star Clusters and the Milky Way, ASP Conf. Ser., 228, 169

Just, A. 2002, Disks of Galaxies: Kinematics, Dynamics and Peturbations, ASP Conf. Ser., 275, 117

Just, A. 2003, Astrophys. Space Sci., 284, 727

Just, A., Fuchs, B., \& Wielen, R. 1996, A\&A, 309, 715

Lequeux, J., Fort, B., Dantel-Fort, M., Cuillandre, J.-C., \& Mellier, Y. 1996, A\&A, 312, L1

Lequeux, J., Combes, F., Dantel-Fort, M., Cuillandre, J.-C., Fort, B., \& Mellier, Y. 1998, A\&A, 334, L9

Lynden-Bell, D. 1975, Vistas Astron., 19, 299

Misiriotis, A., \& Bianchi, S. 2002, A\&A, 384, 866

Misiriotis, A., Kylafis, N. D., Papamastorakis, J., \& Xilouris, E. M. 2000, A\&A, 353,117

Misiriotis, A., Popescu, C. C., Tuffs, R. J., \& Kylafis, N. D. 2001, A\&A, 372, 775

Morrison, H. L., Boroson, T. A., \& Harding, P. 1994, AJ, 108, 1191

Pohlen, M., Dettmar, R.-J., Lütticke, R., \& Aronica, G. 2002, A\&A, 392, 807

Popescu, C. C., Misiriotis, A., Kylafis, N. D., Tuffs, R. J., \& Fischera, J. 2000, A\&A, 362, 138

Rieke, G. H., \& Lebofsky, M. J. 1985, ApJ, 288, 618

Sackett, P. D., Morrison, H. L., Harding, P., \& Boroson, T. A. 1994, Nature, 370, 441

Sancisi, R., \& van Albada, T. S. 1987, ApJ, 295, 305

Sandage, A., \& Walker, M. F. 1966, ApJ, 143,313

Scalo, J. 1986, Fund. Cosmic Phys., 11, 1

Schwarzkopf, U., \& Dettmar, R.-J. 2000, A\&A, 361, 451

Shang, Z., Zheng, Z., Brinks, E., et al. 1998, ApJ, 504, L23

Sofue, Y. 1996, ApJ, 458, 120

Sofue, Y. 1997, PASJ, 49, 17

Stetson, P. B., \& Harris, W. E. 1988, AJ, 96, 909

Stevens, J. A., Amure, M., \& Gear, W. K. 2005, MNRAS, 357, 361

Twarog, B. S. 1980, ApJ, 242, 242

van der Kruit, P. C., \& Searle, L. 1981, A\&A, 95, 116

van der Kruit, P. C., \& Searle, L. 1982a, A\&A, 110, 61

van der Kruit, P. C., \& Searle, L. 1982b, A\&A, 110, 79

Wainscoat, R. J., Freeman, K. C., \& Hyland, A. R. 1989, ApJ, 337, 163

Whittet, D. C. B. 1992, Dust in the Galactic Environment (Bristol: IoP)

Wielen, R. 1977, A\&A, 60, 263

Wielen, R., \& Fuchs, B. 1988, in Lec. Notes in Physics: The Outer Galaxy, 306, 100

Wielen, R., Dettbarn, C., Fuchs, B., Jahreiß, H., \& Radons, G. 1992, The Stellar Populations of Galaxies, IAU Symp., 149, 81

Xilouris, E. M., Kylafis, N. D., Papamastorakis, J., Paleologou, E. V., \& Hserendel, G. 1997, A\&A, 325, 135

Xilouris, E. M., Byun, Y. I., Kylafis, N. D., Paleologou, E. V., \& Papamastorakis, J. 1999, A\&A, 344, 868

Zepf, S. E., Liu, M. C., Marleau, F. R., Sackett, P. D., \& Graham, J. R. 2000, AJ, 119,1701 